\title{
Data Sources and Computational Approaches for Generating Models of Gene Regulatory Networks
}

\author{
B. D. Aguda, ${ }^{1}$ G. Craciun, ${ }^{2}{ }^{\S}$ R. Cetin-Atalay ${ }^{3}$ \\ ${ }^{1}$ Department of Genetics and Genomics, Boston University School of Medicine \\ 715 Albany Street, Boston, Massachusetts, USA 02118 \\ ${ }^{2}$ Mathematical Biosciences Institute, The Ohio State University \\ 231 W. $18^{\text {th }}$ Avenue, Columbus, Ohio, USA 43210 \\ ${ }^{3}$ Department of Molecular Biology \& Genetics, Bilkent University, Ankara, Turkey \\ and Virginia Bioinformatics Institute, Virginia Polytechnic Institute and State \\ University, Blacksburg,Virgina,USA 24061
}


$\S$ This author was supported by the National Science Foundation under

Agreement No. 0112050.

\section{OUTLINE}

Introduction

Formal representation of GRNs

An example of a GRN: The Lac Operon

Hierarchies of GRN models: From probabilistic graphs to mechanistic models

A guide to databases and knowledgebases on the internet

Pathway Databases \& Platforms

Ontologies for GRN modeling

Current Gene, Interaction, and Pathway Ontologies

Whole-cell modeling platforms

Ontology for modeling multi-scale and incomplete networks

An ontology for cellular processes

The PATIKA pathway ontology

Extracting Models from Pathways Databases

Pathway and Dynamic Analysis Tools for GRNs

Global network properties

Recurring network motifs

Identifying pathway channels in networks: extreme pathway analysis

Network stability analysis 
Predicting dynamics \& bistability from network structure alone

Concluding Remarks 


\section{INTRODUCTION}

High-throughput data acquisition technologies in molecular biology, including rapid DNA sequencers, gene expression microarrays and other microchip-based assays, are providing an increasingly comprehensive parts list of a biological cell. Although this parts list may be far from complete at this time, the so-called "post-genomic era" has now begun in which the goal is to integrate the parts and analyze how they interact to determine the system's behavior. This integration is being facilitated by the creation of databases, knowledgebases and other information repositories on the internet. How these huge amounts of information will be used to answer biological questions and predict behavior will keep multidisciplinary teams of scientists busy for many years. A key question is how the expression of genes is regulated in response to various intracellular and external conditions and stimuli. The current paradigm is that the secret to life could be found in the genetic code; however, the expression of genes and the unfolding of the regulatory molecular networks in response to the environment may well be the defining attribute of the living state.

This chapter focuses on gene regulatory networks (GRNs). A "gene regulatory network" refers to a set of molecules and interactions that affect the expression of genes located in the DNA of a cell. Gene expression is the combination of transcription of DNA sequences, processing of the primary RNA transcripts, and translation of the mature messenger RNA (mRNA) to proteins in ribosomes. This picture is often referred to as the "central dogma" and it has been the canonical model for the flow of information from the genetic code to proteins. These processes are shown schematically as steps labeled $\underline{\tau}, \underline{\rho}$ and $\underline{\sigma}$ in Fig 1 . 


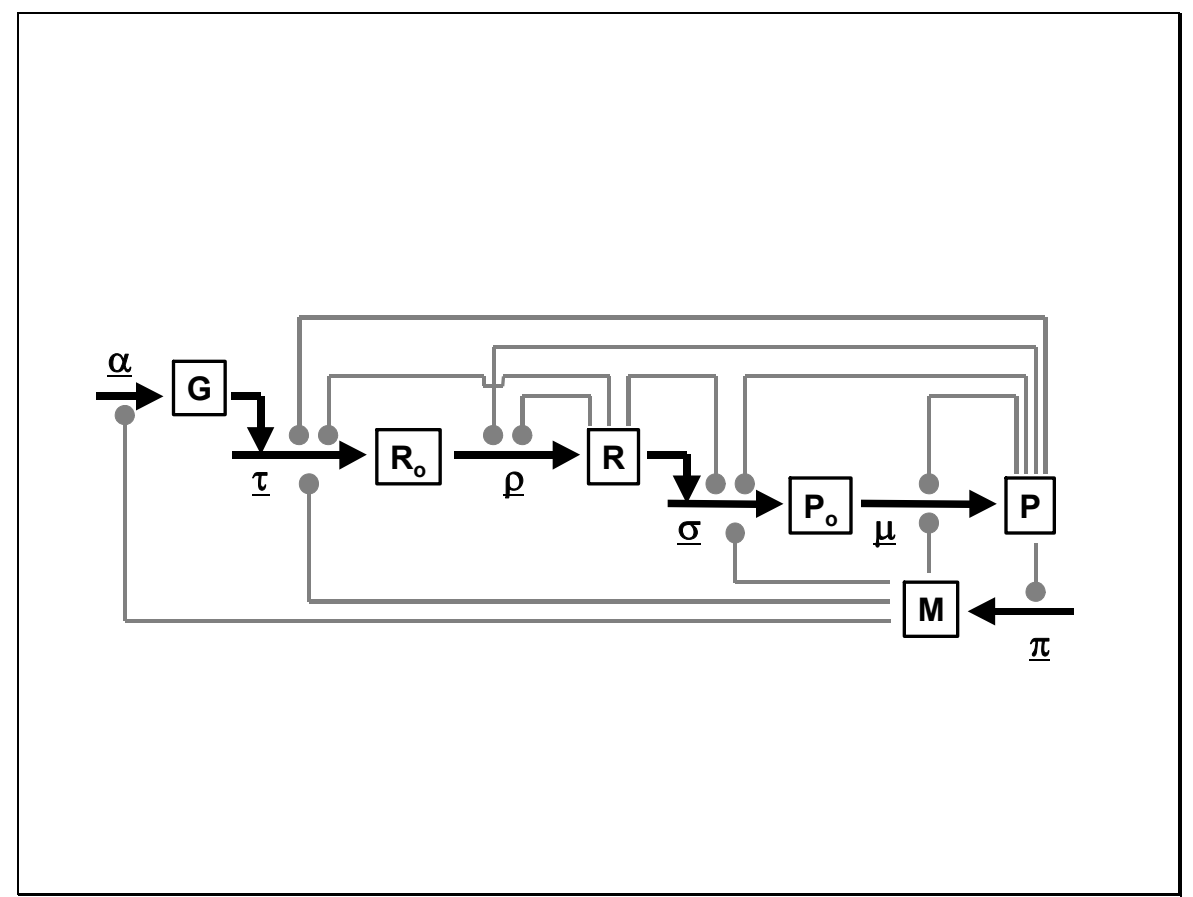

Figure 1. A schematic representation of a gene regulatory network involving modules of molecular classes (shown in boxes); the modules shown are the transcriptional units in the genome $(\mathbf{G})$, primary transcripts $\left(\mathbf{R}_{\mathrm{o}}\right)$, mature transcripts $(\mathbf{R})$, primary proteins $\left(\mathbf{P}_{\mathrm{o}}\right)$, modified proteins $(\mathbf{P})$, and metabolites $(\mathbf{M})$. The labeled steps shown in black lines are transcription $(\underline{\tau})$, RNA processing $(\underline{\rho})$, translation $(\underline{\sigma})$, protein modification $(\underline{\mu})$, metabolic pathways $(\underline{\pi})$, and genome replication $(\underline{\alpha})$. The feedback interactions shown in gray lines are discussed in the text. Filled circles represent either inhibition or activation. 
The step labeled $\underline{\mu}$ in Fig 1 represents modification of primary proteins to render them functional; examples would be post-translational covalent modifications (e.g. phosphorylation) and binding with other proteins or other molecules. Represented within the set of steps $\underline{\mu}$ are the many regulatory events (other than transcription and translation) affecting gene expression and the overall physiology of the cell.

The complexity of GRNs may arise from the many possible feedback loops shown as gray lines in Fig 1. In step $\underline{\tau}$, proteins could be directly involved in transcription, as in the case of transcription factors binding to upstream regulatory regions of genes. Many RNA and protein molecules cooperate in the translation step $\underline{\sigma}$ in Fig 1; examples are tRNA, rRNA, and ribosomal proteins.

The first goal of this chapter is to survey sources of data and other information that can be used to generate models of GRNs. The focus is on biological databases and knowledgebases that are available on the internet, especially those that attempt to integrate heterogeneous information including molecular interactions and pathways. The second goal of this review is to summarize current models of GRNs and how they can be extracted from biological databases. Depending on the nature of the data, different granularities of GRN models can be generated, ranging from probabilistic graphical models to detailed kinetic or mechanistic models. A crucial issue in the design of pathways databases is how to represent information having various levels of uncertainty. Because of its central importance in GRN modeling, an extensive discussion of pathway ontology is given. Lastly, the third goal is to discuss theoretical and computational methods for the analysis of detailed models of GRNs. In particular, a summary is given 
of various tools already developed in the field of reaction network analysis. Particular emphasis of the discussion is on exploiting information on network structure to deduce potential behavior of GRNs without knowing quantitative values of rate parameters.

\section{FORMAL REPRESENTATION OF GRNS}

The GRN of Fig 1 can be formally translated to a set of general dynamical equations. The modules (in boxes) in the GRN represent the following classes of biomolecules:

G : vector of all transcriptional units (TUs) involved in the GRN (in terms, for example, of gene dosage per TU);

$\mathbf{R}_{\mathbf{0}}$ : vector of primary RNA transcripts corresponding to the TUs in G;

R : vector of messenger RNA (mRNA), transfer RNA (tRNA), ribosomal RNA (rRNA), and other processed RNAs;

$\mathbf{P}_{\mathbf{0}}: \quad$ vector of newly translated (primary) proteins;

P : vector of modified proteins;

M : vector of metabolites.

Disregarding the replication of the genomic DNA ( $\operatorname{step} \underline{\alpha}$ ) and the changes in the metabolome $\mathbf{M}$ for now (i.e. assume $\mathbf{G}$ and $\mathbf{M}$ to be constant), a mathematical representation of the dynamics of the GRN in Fig 1 would be the following set of vectormatrix equations: 


$$
\begin{aligned}
& \mathrm{d} \mathbf{R}_{\mathbf{0}} / \mathrm{dt}=\underline{\tau} \mathbf{G}-\underline{\rho} \mathbf{R}_{\mathbf{o}}-\underline{\boldsymbol{\delta}}_{1} \mathbf{R}_{\mathbf{0}} \\
& \mathrm{d} \mathbf{R} / \mathrm{dt}=\underline{\rho} \mathbf{R}_{\mathbf{0}}-\underline{\boldsymbol{\delta}}_{2} \mathbf{R} \\
& \mathrm{d} \mathbf{P}_{\mathbf{o}} / \mathrm{dt}=\underline{\sigma} \mathbf{R}-\underline{\mu} \mathbf{P}_{\mathbf{o}}-\underline{\boldsymbol{\delta}}_{3} \mathbf{P}_{\mathbf{o}} \\
& \mathrm{d} \mathbf{P} / \mathrm{dt}=\underline{\mu} \mathbf{P}_{\mathbf{o}}-\underline{\boldsymbol{\delta}}_{4} \mathbf{P}
\end{aligned}
$$

The "RNA transcription" matrix $\underline{\tau}$ is a diagonal matrix (i.e. all off-diagonal entries are 0 ) with the non-zero entries being, in general, functions of $\mathbf{R}, \mathbf{P}$, and $\mathbf{M}$ as depicted by the feedback loops in Fig 1. The "RNA-processing matrix" $\rho$ is a diagonal matrix with the non-zero entries being, in general, functions of $\mathbf{R}$ and $\mathbf{P}$. The diagonal matrix $\underline{\boldsymbol{\sigma}}$ is called the "protein translation" matrix. The diagonal matrix $\underline{\mu}$ is called the "protein modification" matrix (which includes all post-translational modifications, and proteinprotein interactions). Fig. 1 shows the dependence of $\underline{\boldsymbol{\sigma}}$ and $\underline{\boldsymbol{\mu}}$ to $\mathbf{R}, \mathbf{P}$, and $\mathbf{M}$. The diagonal matrices $\underline{\delta}_{\mathrm{i}}$ are "degradation" matrices which account for the degradation of RNA and protein molecules as well as their transport or dilution. Because of the general dependence of the matrices to the variables $\mathbf{R}, \mathbf{P}$ and $\mathbf{M}$, the above equations are nonlinear equations in these variables.

An example of a GRN is given next to illustrate the formal representation just described. The example also demonstrates the art of modeling and reduction of the network into minimal mathematical models. 


\section{AN EXAMPLE OF A GRN: THE LAC OPERON}

The lac operon in the bacterium Escherichia coli is a well-studied GRN. This

prokaryotic gene network has been the subject of numerous reviews; ${ }^{1-4}$ it is discussed here primarily to illustrate the various aspects of GRN modeling, starting with the information on genome organization (operon structure) to knowledge on protein-DNA interactions, protein-protein interactions and the influence of metabolites.

Understanding the lac operon begins by looking at the genome organization of $E$. coli. The complete genome sequence of various strains of this bacterium can be accessed through the webpage of the National Center for Biotechnology Information (http://www.ncbi.nlm.nih.gov). From the homepage menu, clicking Entrez followed by Genome gives the link to complete bacterial genomes including E. coli. Genes in the circular chromosome of $E$. coli are organized into 'operons'. An operon is a cluster of genes whose expression is controlled by a common set of operator sequences and regulatory proteins. ${ }^{5}$ The genes in the cluster are usually involved in the synthesis of enzymes needed for the metabolism of a molecule. Several reviews on the influence of operon structure on the dynamical behavior of GRNs are available. ${ }^{6-7}$

The lac operon is shown in Fig 2A. The GRN involves the gene set $\{l a c Z, \operatorname{lac} Y, \operatorname{lac} A, \operatorname{lac} I\}$ and the regulatory sequences $\{\mathrm{O} 1, \mathrm{O} 2, \mathrm{O} 3, \mathrm{~A}\}$ as shown in Fig 2A. The gene lacI encodes a repressor protein that binds the operator sequences $\mathrm{O} 1$, 


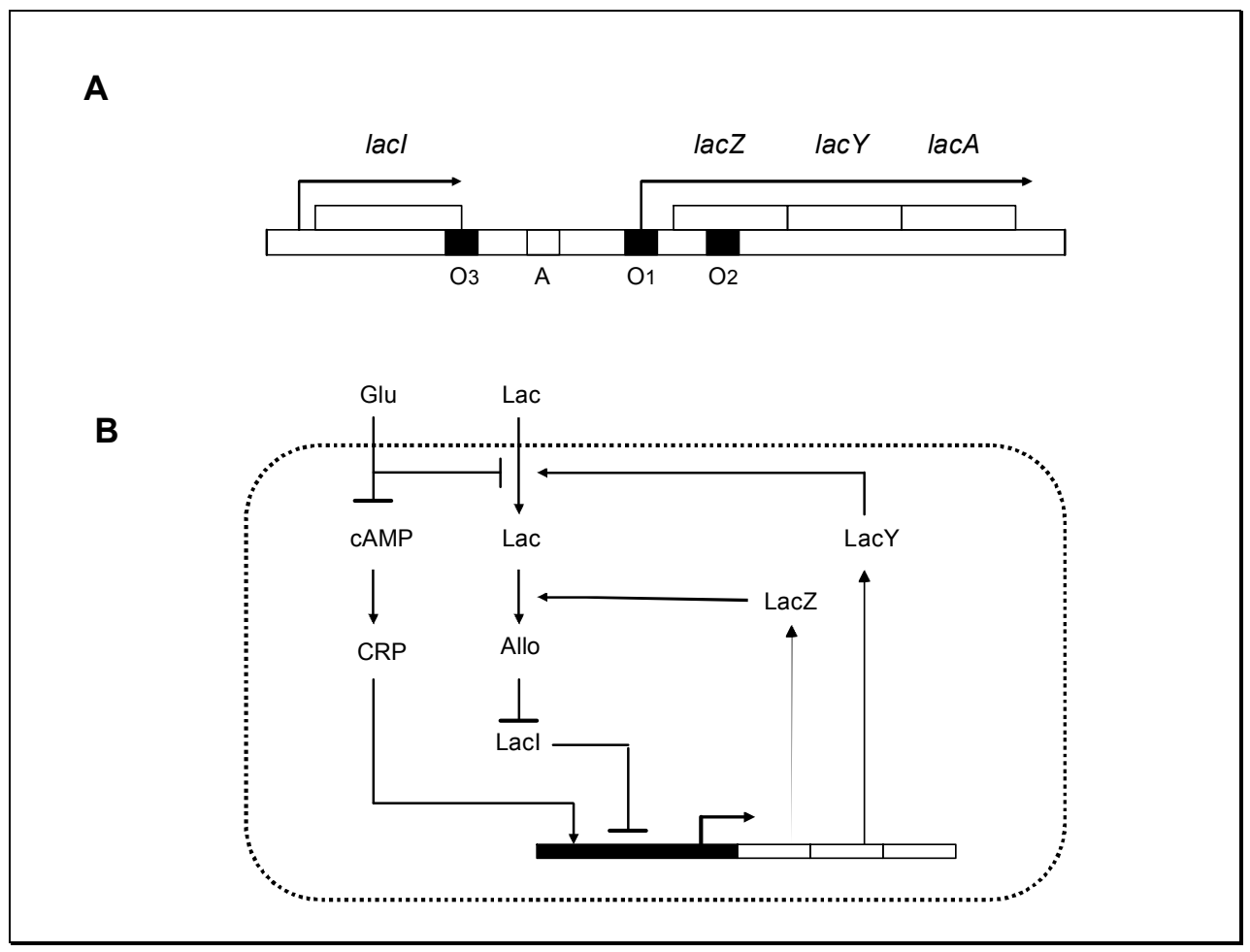

Figure 2. The Lac Operon. (A) The expression of the genes $l a c Z$, lacY and lacA as one transcriptional unit is controlled by the upstream regulatory sequences including the operator regions $\mathrm{O} 1, \mathrm{O} 2$ and $\mathrm{O} 3$ where the repressor protein (the product of the lacI gene) binds. The CRP/cAMP protein complex binds the sequence A resulting in increased transcription. (B) A schematic representation of the key pathways regulating the Lac Operon. (Figure is modified from Ref. 3). 
$\mathrm{O} 2$, and $\mathrm{O} 3$ thereby repressing the synthesis of the lacZ-lacY-lacA transcript. Gene lacZ encodes the $\beta$-galactosidase enzyme, gene $l a c Y$ encodes a permease, and gene $\operatorname{lac} A$ encodes a transacetylase. The CRP/cAMP complex binds the sequence A and enhances transcription.

The key pathways that generate the switching behavior of the GRN are shown in Fig 2B. This switching behavior of the lac operon explains the diauxic growth (shift from glucose to lactose utilization) of $E$. coli. If there is glucose in the growth medium, the operon is always OFF because glucose inhibits cAMP and lactose transport into the cell. If glucose is absent, the operon would remain OFF unless some lactose is present inside the cell (which is true when glucose is depleted and lactose from the outside can now enter the cell); an initially small amount of internal lactose increases rapidly due to at least two positive feedback loops as shown in Fig 2B. It is the positive feedback loop involving lactose transport that ultimately controls the influx of lactose.

In terms of the formal representation of the lac operon according to Fig 1, the vectors of variables corresponding to the model shown in Fig 2B are the following: $\mathbf{G}=\left[\begin{array}{lll}G_{Z Y A} & G_{I} & G_{C R P}\end{array}\right]^{T}$ where $G_{Z Y A}$ is the base sequence on DNA that includes genes $l a c Z$, lacY, and $l a c A$, and transcribed as one transcriptional unit ([ ] $]^{\mathrm{T}}$ means 'transpose'); $\mathrm{G}_{\mathrm{I}}$ is the DNA sequence containing gene $l a c I$, and $\mathrm{G}_{\mathrm{CRP}}$ is the transcribed DNA sequence containing gene $C R P . \mathbf{R}_{\mathbf{o}}=\left[\begin{array}{lll}\mathrm{R}_{\mathrm{ZYA}, \mathrm{o}} & \mathrm{R}_{\mathrm{I}, \mathrm{o}} & \mathrm{R}_{\mathrm{CRP}, \mathrm{o}}\end{array}\right]^{\mathrm{T}}$ is the vector of primary transcripts; $\mathbf{R}=\left[\begin{array}{llll}R_{Z} & R_{Y} & R_{I} & R_{C R P}\end{array}\right]^{T}$ is the vector of mature transcripts. Note that the transcript $\mathrm{R}_{\mathrm{A}}$ (corresponding to gene $\mathrm{A}$ ) is not included because it is not considered further in the dynamics of the GRN. $\mathbf{P}_{\mathbf{o}}=\left[\begin{array}{llll}\mathrm{P}_{Z, \mathrm{o}} & \mathrm{P}_{\mathrm{Y}, \mathrm{o}} & \mathrm{P}_{\mathrm{I}, \mathrm{o}} & \mathrm{P}_{\mathrm{CRP}, \mathrm{o}}\end{array}\right]^{\mathrm{T}}$ is the vector of primary protein 
translates; $\mathbf{P}=\left[\begin{array}{llll}\mathrm{P}_{4 Z} & \mathrm{P}_{\mathrm{Ym}} & \mathrm{P}_{4 \mathrm{I}} & \mathrm{P}_{\mathrm{CRP} . c A M P}\end{array}\right]^{\mathrm{T}}$ is the vector of mature, modified, and active proteins; the protein $\mathrm{P}_{\mathrm{Z}}$ ( $\beta$-galactosidase) is tetrameric in its functional form, the permease $\mathrm{P}_{\mathrm{Y}}$ acts at the plasma membrane (hence the subscript ' $\mathrm{m}$ ' in $\mathrm{P}_{\mathrm{Ym}}$ ), the repressor protein $\mathrm{P}_{\mathrm{I}}$ is tetrameric, and CRP's binding with cAMP is necessary for its DNA-binding activity. $\mathbf{M}=\left[\begin{array}{llll}\text { Glu Lac Allo } & \mathrm{cAMP}\end{array}\right]^{\mathrm{T}}$ is the vector of metabolites $(\mathrm{Glu}=$ glucose, Lac $=$ lactose, Allo $=$ allolactose, $\mathrm{cAMP}=$ cyclic adenosine monophosphate $).$ The GRN for the lac operon model using the representation of Fig 1 is shown in Fig 3. The first equation in [1] would look like this:

$$
\left(\begin{array}{l}
\mathrm{dR}_{\mathrm{ZYA}, \mathrm{o}} / \mathrm{dt} \\
\mathrm{dR}_{\mathrm{l}, \mathrm{o}} / \mathrm{dt} \\
\mathrm{dR}_{\mathrm{CRP}, \mathrm{o}} / \mathrm{dt}
\end{array}\right)=\left(\begin{array}{ccc}
\tau_{11} & 0 & 0 \\
0 & \tau_{22} & 0 \\
0 & 0 & \tau_{33}
\end{array}\right)\left(\begin{array}{l}
\mathrm{G}_{\mathrm{ZYA}} \\
\mathrm{G}_{\mathrm{I}} \\
\mathrm{G}_{\mathrm{CRP}}
\end{array}\right)-\left(\begin{array}{ccc}
\rho_{11} & 0 & 0 \\
0 & \rho_{22} & 0 \\
0 & 0 & \rho_{33}
\end{array}\right)\left(\begin{array}{l}
\mathrm{R}_{\mathrm{ZYA}, \mathrm{o}} \\
\mathrm{R}_{\mathrm{I}, \mathrm{o}} \\
\mathrm{R}_{\mathrm{CRP}, \mathrm{o}}
\end{array}\right)-\left(\begin{array}{ccc}
\delta_{11} & 0 & 0 \\
0 & \delta_{22} & 0 \\
0 & 0 & \delta_{33}
\end{array}\right)\left(\begin{array}{l}
\mathrm{R}_{\mathrm{ZYA}, \mathrm{o}} \\
\mathrm{R}_{\mathrm{l}, \mathrm{o}} \\
\mathrm{R}_{\mathrm{CRP}, \mathrm{o}}
\end{array}\right)
$$

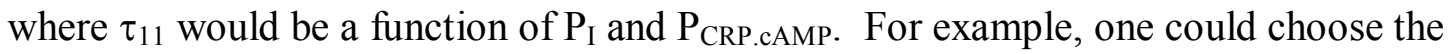
function $\tau_{11}=\left(c_{1}+c_{2} P_{C R P . c A M P}\right) /\left(c_{3}+c_{4} P_{I}^{n}\right)$ to represent the activation of transcription by the protein complex $\mathrm{P}_{\text {CRP.cAMP }}$ and inhibition by the tetrameric repressor $\mathrm{P}_{\mathrm{I}}$ (the $\mathrm{n}$ and $\mathrm{c}_{\mathrm{i}}$ 's are constant parameters; $\mathrm{n}$ should be greater than 1 because of the tetrameric complex of $\left.\mathrm{P}_{\mathrm{I}}\right)$. 


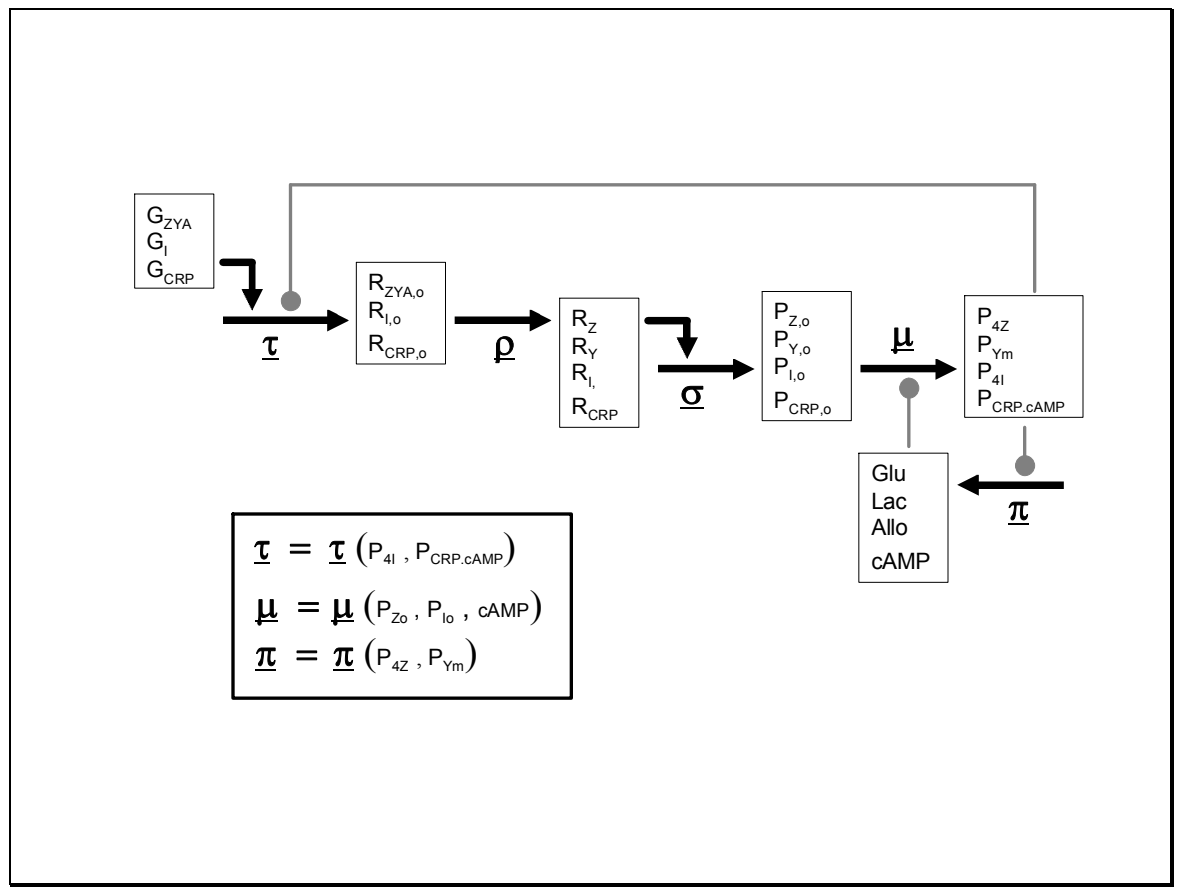

Figure 3. The Lac Operon in accordance with the scheme shown in Fig 1. See text for details. 
New mathematical models and reviews on the lac operon have appeared recently. ${ }^{2-4}$ Yildirim and Mackey ${ }^{2}$ used delay differential equations to account for the transcriptional and translational steps that are missing in their model. An earlier detailed kinetic model was proposed and analyzed by Wong, Gladney and Keasling. ${ }^{1}$ Recently, Vilar, Guet, and Leibler ${ }^{4}$ used a 4-variable model that captures many of the essential dynamics of the lac operon. Note that the Vilar-Guet-Leibler model is essentially a threevariable model. The bistability exhibited by the model was used as the explanation for the ON-OFF behavior of the lac operon.

At the single-cell level, the operon is either ON or OFF (all-or-nothing induction) as shown in the recent experimental report of Ozbudak et al. ${ }^{3}$ These authors exploited the positive feedback loop between the permease (y) and the inducer (x), and used the following mathematical model to represent the positive feedback loop:

$$
\begin{aligned}
\tau_{\mathrm{y}} \mathrm{dy} / \mathrm{dt} & =\alpha\left(1 /\left[1+\mathrm{R} / \mathrm{R}_{\mathrm{o}}\right]\right)-\mathrm{y} \\
\tau_{\mathrm{x}} \mathrm{dx} / \mathrm{dt} & =\beta \mathrm{y}-\mathrm{x}
\end{aligned}
$$

where $\mathrm{R} / \mathrm{R}_{\mathrm{T}}=1 /\left[1+\left(\mathrm{x} / \mathrm{x}_{\mathrm{o}}\right)^{\mathrm{n}}\right]$

and $\mathrm{R}=$ concentration of active $\mathrm{LacI}, \mathrm{R}_{\mathrm{o}}=$ initial concentration of active $\mathrm{LacI}, \mathrm{R}_{\mathrm{T}}=$ total concentration of LacI tetramers, $x_{0}=$ initial concentration of LacY (permease), and the rest of the symbols are parameters. The parameter $n$ allows consideration of the fact that the repressor is a tetramer. This simple model generates bistability in which the all-ornothing transition is associated with a saddle-node bifurcation. The simple set of equations above was useful in guiding the authors' experiments in showing ON-OFF 
behavior as well exploring the phase diagram (coordinates of which are the variables $\mathrm{x}$ and $\mathrm{y}$, for example) for bistable and monostable regions.

The lac operon illustrates several important points in modeling GRNs. Although the operon structure is not a general property of all genomes, one can expect that genomic DNA sequence organization affects the dynamics of the GRN; this is primarily due to co-expression of genes found in the same transcriptional units or co-regulation of genes by transcription factors that recognize promoter regions having similar regulatory sequences. Another lesson from the lac operon is that abstraction of the complex GRN may be sufficient to understand the behavior of the system. This abstraction was facilitated by prior knowledge of the influence of network topology on dynamical behavior, e.g. bistability arising from positive feedback loops. ${ }^{8}$ A discussion on how network structure alone influences system behavior is provided in the penultimate section of this chapter.

\section{HIERARCHIES OF GRN MODELS: FROM PROBABILISTIC GRAPHS TO DETERMINISTIC MODELS}

The general representation of GRNs in Fig 1 considers groups of molecules according to their chemical classes (DNA, RNA, proteins, metabolites) whose "interactions" merely encode the broad concepts of transcription, post-transcriptional processing, translation and post-translational modifications. Depending on the nature of 
available experimental information, specific models of gene regulatory networks can be constructed at various levels of detail.

Networks, in general, are described by their graphical structures. A graph is basically a set of 'nodes' and a set of 'edges', the latter being the representations of the interactions or associations among nodes. Progressively more detailed mechanistic information can be added to a graph as they become available. At one extreme of the spectrum of models, the nodes in the graph could be just a set of genes (and no other kinds of objects), with certain pairs of genes linked by undirected edges if these pairs are known to "interact" or are "associated" in some way. Sometimes the nodes could be proteins and the edges represent physical interactions. Because of the correspondence between proteins and genes (albeit not generally one-to-one), protein-protein interaction networks may imply some underlying GRN structure. In general, nodes in a graph can be defined according to the level of detail that is sufficient to describe a particular feature, function or behavior of the system. For example, the nodes in Fig 1 represent various classes of molecules. A node could also represent a subnetwork or module with specific cellular function.

An edge of a graph is assigned a direction if there is information on causality, i.e. that one node affects the state of the other. A directed edge can be further characterized as either "activating" or "inhibiting". As more quantitative data are available, it may be possible to identify the "strength" of an edge. For dynamic models, the strength of an edge would, for example, require identification of rate expressions as functions of the states of the nodes. At this point, a dynamic model encoded in deterministic differential equations is possible. Finally, at the other extreme in the spectrum of GRN models, 
microscopic details of the interactions between individual molecular species are known and molecular dynamic simulations are possible.

As the example of the lac operon illustrates, abstract models involving differential equations that do not necessarily reflect the detailed mechanism are sometimes used when the goal is primarily to explore possible system dynamics arising from the structure of the network. Associated with the process of 'abstraction' is the problem of reducing the network into a smaller set of 'modules' and their interactions. Modules can range from individual molecules or genes, to a set of genes or proteins, or to functional subnetworks with definable cellular functions. Similar ideas have been discussed recently by Vilar et al. ${ }^{4}$ in their work on the lac operon. The lac operon is an example of a well-defined small model system in which a considerable amount of biological knowledge and mechanistic understanding have already accumulated so that refined mathematical modeling can be carried out. Many other focused models and corresponding mathematical model formalisms have been reviewed recently by de Jong. ${ }^{9}$ In contrast, constructing the network graph of gene interactions from large-scale gene expression measurements is just beginning and is, at times, controversial. Since this field has been reviewed ${ }^{10-13}$ recently only a brief account is given below.

High-throughput gene expression measurements using DNA microarrays provide global snapshots of the dynamics of gene networks at the RNA level. Expression data are intrinsically noisy and conclusions derived from them are probabilistic in nature. Furthermore, the mRNA levels are averages from cell populations. Gene network reconstruction from microarray data also suffers from the so-called 'dimensionality problem, ${ }^{11}$ because the number of genes is much greater than the number of microarray 
experiments. Statistical analysis of gene expression data usually employ clustering methods to find genes with similar expression patterns across time series or across different experimental conditions (e.g. see Refs. 14-15). The assumption is that clustered or co-expressed genes are somehow co-regulated or perhaps share similar functions. The results of clustering in terms of GRN modeling could therefore be a coarse-grain network composed of modules (nodes), each module representing a set of genes with similar functions.

Graphical models that combine probability theory and graph theory are suitable frameworks for inferring GRNs from gene expression data. ${ }^{10,16}$ In general, these graphical models are probability models for multivariate random variables whose independence structure can be represented by a conditional independence graph. Recently, Friedman ${ }^{10}$ reviewed the field of probabilistic graphical models for gene networks, including Bayesian networks. In a Bayesian network, the nodes represent random variables (e.g. genes and their expression levels) while the edges show conditional dependence relations. Husmeier ${ }^{17-18}$ has also reviewed the applications of Bayesian networks to microarray data. Bayesian networks were first applied to the problem of reverse engineering of GRNs from microarray expression data by Friedman et al., ${ }^{19}$ Pe'er et al., ${ }^{20}$ and Hartemink et al. ${ }^{21}$ Other examples of graphical models employing various statistical methods are discussed by Wang, Myklebost and Hovig. ${ }^{16}$

Zak et al. ${ }^{22}$ have argued that inferring the GRN structure from expression data alone is impossible. However, promising results come from more recent work showing that properly designed perturbation experiments do permit network reconstruction (see Refs. 12, 13, 18, 23-25). Two papers ${ }^{23-24}$ extended ideas from metabolic control analysis 
to suggest perturbation experiments designed to determine the direction and strengths of interactions between genes. Also, Gardner et al. ${ }^{25}$ used systematic perturbations combined with least-squares regression to infer the gene network topology and weights of interactions.

In general, the issues encountered during the creation of a GRN graph are similar to those faced when designing a pathway or interaction database. These issues will be discussed in more detail in the section on 'pathway ontology' below. An extensive discussion on this ontology is provided because it is a crucial stepping stone for future projects concerned with the extraction of GRN models from pathways databases. Pathways databases are relatively recent developments in bioinformatics. These databases are built from more elementary databases and it is important to be aware of the many heterogeneous bioinformatics resources available, most of them on the internet. Thus, a brief guide is given next.

\section{A GUIDE TO DATABASES AND KNOWLEDGEBASES ON THE INTERNET}

The field of bioinformatics has naturally arisen to cope with the deluge of data generated by high-throughput technologies in genomics, transcriptomics, proteomics, and other -omics. These data are organized into databases (DBs) and knowledgebases (KBs), many of which are publicly available on the internet. Comprehensive and realistic modeling of GRNs should tap into the information contained in these DBs and KBs. Thus, it is expected that the next generation of modelers will have to be sufficiently 
aware of bioinformatics resources. It is for this reason that an overview of the major bioinformatics DBs and KBs is provided here, although their utility for modeling GRNs may not be direct and obvious at this time. It was alluded to in the discussion of the lac operon that understanding the operon structure of the genomic DNA was necessary to understand the dynamics of the network. In general, relating genome organization to GRN dynamics is a very difficult and still a very much open problem. This section begins with genomic sequence databases in anticipation of their future use in helping predict GRN structures; a specific example would be that of finding regulatory sequences where transcription factors bind thereby linking one gene product to the transcription of another gene.

To date, the genomes of more than 150 organisms have been sequenced, and many more sequencing projects are currently going on or planned. Publicly available DNA sequence data as well as functional and structural data on proteins are accumulating at an exponential rate, virtually doubling every year. The major sequence and structure repositories which are regularly updated are listed in Table 1. 
Table 1. Major sequence and structure repositories

\begin{tabular}{|l|l|l|}
\hline Database & Description & URL \\
\hline \hline GenBank & $\begin{array}{l}\text { Repository of all publicly available annotated } \\
\text { nucleotide and protein sequences }\end{array}$ & http://www.ncbi.nlm.nih.gov/ \\
\hline EMBL Database & $\begin{array}{l}\text { Repository of all publicly available annotated } \\
\text { nucleotide and protein sequences }\end{array}$ & http://www.ebi.ac.uk/embl.html \\
\hline $\begin{array}{l}\text { DDBJ (DNA Data } \\
\text { Bank of Japan) }\end{array}$ & $\begin{array}{l}\text { Repository of all publicly available annotated } \\
\text { nucleotide and protein sequences }\end{array}$ & http://www.ddbj.nig.ac.jp \\
\hline PIR & $\begin{array}{l}\text { Protein information resource: protein sequence } \\
\text { database }\end{array}$ & http://pir.georgetown.edu/ \\
\hline Swiss-Prot & $\begin{array}{l}\text { Highly annotated curated protein sequence database } \\
\text { available 3D structures of proteins and nucleic acids }\end{array}$ & http://www.expasy.org/sprot \\
\hline PDB & http://www.rcsb.org/pdb \\
\hline
\end{tabular}

Table 2. Protein sequence and structure property databases

\begin{tabular}{|c|c|c|}
\hline Database & Description & URL \\
\hline eMOTIF & Protein sequence motif database & http://motif.stanford.edu/emotif \\
\hline InterPro & Integrated resource of protein families, domains & http://www.ebi.ac.uk/interpro \\
\hline iProClass & Integrated protein classification database & http://pir.georgetown.edu/iproclass/ \\
\hline ProDom & Protein domain families & http://www.toulouse.inra.fr/prodom.html \\
\hline $\mathrm{CDD}$ & $\begin{array}{l}\text { Conserved domain database: covers protein domain } \\
\text { information from Pfam, SMART and COG databases }\end{array}$ & $\begin{array}{l}\text { http://www.ncbi.nlm.nih.gov/Structure/cdd } \\
\text { /cdd.shtml }\end{array}$ \\
\hline CATH & Protein structure classification database & http://www.biochem.ucl.ac.uk/bsm/cath/ \\
\hline $\mathrm{CE}$ & Repository of 3D Protein structure alignments & http://cl.sdsc.edu/ce.html \\
\hline SCOP & Structural classification of proteins & http://scop.mrc-lmb.cam.ac.uk/scop \\
\hline
\end{tabular}


The partners of the International Nucleotide Sequence Databases (INSD), namely GenBank, EMBL and $D D B J$, share their nucleic acid sequence data for a comprehensive coverage of all available genome information. Swiss-Prot is a manually curated protein sequence database with a high level annotation of protein function and protein modifications, including links to property, structure and pathways databases. PIR is similar to Swiss-Prot, with the former providing some options for sequence analysis. Recently, UniProt Knowledgebase (http://www.uniprot.org) was established with the aim of unifying and linking protein databases with cross-references and query options.

Some of the major protein sequence and structure property databases are listed in Table 2. Although there are many more general or specialized property databases available, ${ }^{26}$ the list given in Table 2 is a good start for exploring protein property databases. Table 3 gives a list of gene expression repositories.

It is very difficult for one person to keep up with the rapidly increasing number of genomics, proteomics, and interactomics and metabolomics databases, let alone their intended usage. ${ }^{26}$ To alleviate this problem, an increasing number of integrated database retrieval and analysis systems tools are being developed for the purpose of data management, acquisition, integration, visualization, sharing and analysis. Table 4 lists promising examples of these tools, which are regularly maintained and updated. GeneCards is an integrated database of human genes, genomic maps, proteins, and diseases, with software that retrieves, combines, searches, and displays human genome information. GenomNet is of particular interest since its analytical tools are 
Table 3. Gene expression databases

\begin{tabular}{|l|l|l|}
\hline Database & Description & URL \\
\hline \hline ArrayExpress & Microarray gene expression data collection database & $\mathrm{http} / /$ www.ebi.ac.uk/arrayexpress \\
\hline CIBEX & $\begin{array}{l}\text { Center for Information Biology gene: a public } \\
\text { repository for high-throughput experimental data in } \\
\text { gene expression }\end{array}$ & $\mathrm{http} / /$ cibex.nig.ac.jp \\
\hline GeneNote & $\begin{array}{l}\text { Database of human genes expression profiles in } \\
\text { healthy tissues }\end{array}$ & $\mathrm{http} / /$ genecards.weizmann.ac.il/genenote/ \\
\hline GEO & $\begin{array}{l}\text { Gene Expression Omnibus: a high-throughput gene } \\
\text { expression data repository }\end{array}$ & $\mathrm{http} / /$ ncbi.nlm.nih.gov/geo \\
\hline SMD & $\begin{array}{l}\text { Stanford Microarray Database; Raw and normalized } \\
\text { data from microarray experiments }\end{array}$ & $\begin{array}{l}\mathrm{http} / / \text { genome- } \\
\text { www.stanford.edu/microarray }\end{array}$ \\
\hline
\end{tabular}

Table 4. Integrated database retrieval and analysis systems

\begin{tabular}{|c|c|c|}
\hline Database & Description & URL \\
\hline GeneCards & $\begin{array}{l}\text { Database of human genes, proteins and their involvement } \\
\text { in diseases }\end{array}$ & http://bioinfo.weizmann.ac.il/cards \\
\hline GenomeNet & $\begin{array}{l}\text { Network of database and computational services for } \\
\text { genome research }\end{array}$ & http://www.genome.ad.jp/ \\
\hline NCBI & Retrieval system for searching several linked databases & http://www.ncbi.nlm.nih.gov \\
\hline PathPort/ToolBus & $\begin{array}{l}\text { Collection of web-services for gene prediction and } \\
\text { multiple sequence alignment, along with visualization } \\
\text { tools }\end{array}$ & https://www.vbi.vt.edu/pathport \\
\hline SRS-EBI & $\begin{array}{l}\text { Integration system for both data retrieval and applications } \\
\text { for data analysis }\end{array}$ & http://srs.ebi.ac.uk \\
\hline
\end{tabular}


tightly linked with the KEGG pathways database (discussed in the next section). ToolBus comprises several data analysis software platforms such as multiple sequence alignment, phylogenetic trees, generic XML viewer, pathways and microarray analysis, which are linked to each other as well as to major databases. $S R S$ and $N C B I$ serve as general data retrieval portals as well as to provide links to specific analysis tools.

\section{PATHWAYS DATABASES AND PLATFORMS}

Along with recent advances in genomics and proteomics, requirements for analysis, expansion and visualization of cell signaling, GRNs and protein-protein interaction maps are leading to the development of data representation and integration tools. Pathways databases can be classified into four groups according to their interactome data content and representation as listed in Table 5. Only those websites that are regularly maintained are included in the list. The first group of databases represents binary interaction databases. BIND, DIP, and MINT document experimentally determined protein-protein interactions from peer-reviewed literature or from other curated databases. BIND and MINT store experimental conditions used to observe the interaction, chemical action, kinetics and other information linked to the original research articles.

Static image databases are very good sources of pathway diagrams which provide a broad introductory view of cell regulatory pathways along with good reviews and links. 
Table 5. Pathways databases and platforms

\begin{tabular}{|c|c|c|c|}
\hline & Database & Description & URL \\
\hline \multirow{7}{*}{ 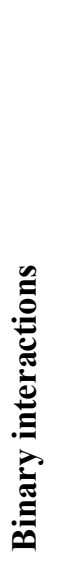 } & BIND & Biomolecular interaction network database & http://www.bind.ca \\
\hline & BindingDB & $\begin{array}{l}\text { Collection on experimental data on the noncovalent } \\
\text { association of molecules in solution }\end{array}$ & http://www.bindingdb.org \\
\hline & BRENDA & $\begin{array}{l}\text { Enzyme Information System: sequence, structure, } \\
\text { specificity, stability, reaction parameters, isolation } \\
\text { data and molecular functions ontology }\end{array}$ & http://www.brenda.uni-koeln.de \\
\hline & DIP & Database of interacting proteins & http://dip.doe-mbi.ucla.edu \\
\hline & IntAct project & $\begin{array}{l}\text { Public repository for annotated protein-protein } \\
\text { interaction data }\end{array}$ & http://www.ebi.ac.uk/intact \\
\hline & InterDom & $\begin{array}{l}\text { Putative interacting protein domain database derived } \\
\text { from multiple sources }\end{array}$ & http://interdom.lit.org.sg \\
\hline & MINT & A molecular interaction database & http://mint.bio.uniroma2.it/mint/ \\
\hline \multirow{3}{*}{ 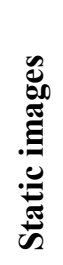 } & ACSF & Signaling resource for signal transduction elements & http://www.signaling-gateway.org/ \\
\hline & BioCarta & $\begin{array}{l}\text { Molecular relationship map pages from areas of } \\
\text { active research }\end{array}$ & http://www.biocarta.com \\
\hline & STKE & Signal transduction knowledge environment & http://stke.org/ \\
\hline \multirow{4}{*}{ 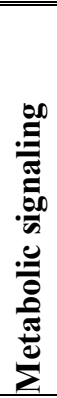 } & BRITE & $\begin{array}{l}\text { Biomolecular relations in information transmission } \\
\text { and expression }\end{array}$ & http://www.genome.ad.jp/brite \\
\hline & KEGG & $\begin{array}{l}\text { Kyoto encyclopedia of genes and genomes: } \\
\text { molecular interaction networks of metabolic and } \\
\text { regulatory pathways }\end{array}$ & http://www.genome.ad.jp/kegg \\
\hline & BioCyc & $\begin{array}{l}\text { A collection of databases that describes the genome } \\
\text { and metabolic pathways of a single organism }\end{array}$ & http://biocyc.org/ \\
\hline & PathDB & $\begin{array}{l}\text { A data repository and a system for building and } \\
\text { visualizing cellular networks }\end{array}$ & http://www.ncgr.org/pathdb \\
\hline \multirow{6}{*}{ 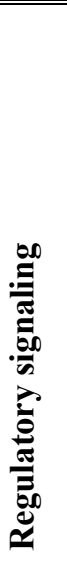 } & aMAZE & $\begin{array}{l}\text { A system for the representation, annotation, } \\
\text { management and analysis of biochemical and gene } \\
\text { regulatory networks }\end{array}$ & http://www.amaze.ulb.ac.be/ \\
\hline & Cytoscape & $\begin{array}{l}\text { Software platform for visualizing molecular } \\
\text { interaction networks }\end{array}$ & http://www.cytoscape.org/ \\
\hline & GeneNet & $\begin{array}{l}\text { Database on gene network components and a } \\
\text { program for the data visualization. }\end{array}$ & $\begin{array}{l}\text { http://wwwmgs.bionet.nsc.ru/mgs/gnw/gene } \\
\text { net }\end{array}$ \\
\hline & PATIKA & $\begin{array}{l}\text { Software platform for pathway analysis tool for } \\
\text { integration and knowledge acquisition }\end{array}$ & http://www.patika.org/ \\
\hline & PathwayAssist & $\begin{array}{l}\text { Tool for analysis, expansion and visualization of } \\
\text { biological pathways, gene regulation networks and } \\
\text { protein interaction maps }\end{array}$ & $\begin{array}{l}\text { http://www.ariadnegenomics.com/products/ } \\
\text { pathway.html }\end{array}$ \\
\hline & TRANSPATH & $\begin{array}{l}\text { Gene regulatory network and microarray analysis } \\
\text { system. }\end{array}$ & $\begin{array}{l}\text { http://www.biobase.de/pages/products/datab } \\
\text { ases.html }\end{array}$ \\
\hline
\end{tabular}


ACSF, STKE and Biocarta are comprehensive knowledgebases on signal transduction pathways and other regulatory networks.

Metabolic signaling databases contain detailed information on metabolic pathways. These DBs have well established data structures but have non-uniform ontologies. BioCyc is a collection of pathway/genome databases for many bacteria and up to 14 species of other organisms. Enzyme catalyzed reactions, or the gene that encodes that enzyme or the structures of chemical compounds in pathways and reactions, can be displayed by BioCyc ontology based software for a given biochemical pathway. In addition $B i o C y c$ supports computational tools for simulation of metabolic pathways.

$K E G G$ is a frequently (daily) updated group of databases for the computerized knowledge representation of molecular interaction networks in metabolism, genetic information processing, environmental information processing, cellular processes and human diseases. The data objects in the $K E G G$ databases are all represented as graphs and various computational methods for analyzing and manipulating these graphs are available.

The fourth category of the DBs and software platforms listed in Table 5 is concerned with regulatory signaling networks. GeneNet, aMAZE and PATIKA possess very similar ontologies for representing and analyzing molecular interactions and cellular processes. PATIKA and GeneNet provide graphical user interfaces for illustrating signaling networks. The aMAZE tool called LightBench ${ }^{27}$ allows users to browse information stored in the database which covers chemical reactions, genes and enzymes involved in metabolic pathways, and transcriptional regulation. Another $a M A Z E$ tool called SigTrans is a database of models and information of signal transduction pathways. 
Both GeneNet and PATIKA are composed of a server-side with a database and client-side. In addition to its database components, a PATIKA client-side editor software provides an integrated, multi-user environment for visualizing, entering and manipulating networks of cellular events independent of an additional web-browser.

Cytoscape and PathwayAssist are similar software tools for automated analysis, integration and visualization of protein interaction maps. In these tools, automated methods for mining PubMed and other public literature databases are incorporated to facilitate the discovery of possible interactions or associations between genes or proteins.

\section{ONTOLOGIES FOR GRN MODELING}

Bioinformatics is now moving towards the direction of creating tools, languages and software for the integration of heterogeneous biological data and their analysis at the level of cellular systems and beyond. This direction requires establishing appropriate 'ontologies' to annotate the various parts and events occurring in the system. An ontology is a set of controlled and unambiguous vocabulary for describing objects and concepts. $^{28}$

Current Gene, Interaction, and Pathway Ontologies

At the genome level, the Gene Ontology ${ }^{\mathrm{TM}}(G O)$ Consortium (http://www.geneontology.org) introduced a comprehensive bio-ontology that is aimed to cover genes in all organisms. $G O$ provides unique identifiers for each concept related to 
"molecular function", "biological process" and "cellular component" searchable through the $A m i G O$ tool (http://www.godatabase.org). Note that these three concepts (especially the concept of "biological process") can be interpreted in terms of memberships of genes in cellular pathways; hence $G O$ can be considered as part of a pathway ontology.

A conventional approach for representing cellular pathways is the use of static diagrams such as those found in the websites of ACSF, BioCarta and STKE (see Table 5). These diagrams are often not reusable, and the pathway representations are far from being uniform and consistent among different websites; this is because the various representations carry implicit conventions rather than explicit rules as required by formal ontologies. Because pathways are basically composed of components and steps or processes, the development of interaction databases is a logical first step (see sample databases in Table 5-Binary interactions). These databases provide diverse amount of binary interaction data, which could then be used for building networks.

Among the cellular pathways, metabolic pathways are generally more detailed and structured because of more advanced knowledge about metabolism in cells (see Table 5-Metabolic signaling). In all of these databases, the proteins are classified according to the Enzyme Commission list of enzymes (EC numbers). These metabolic DBs have strict ontologies which are focused on protein activities relevant to metabolic pathways. Due to a detailed knowledgebase and ontology, metabolic pathways are quite amenable to kinetic modeling and computer simulations. ${ }^{29}$ 


\section{Whole-cell modeling platforms}

There are a number of whole-cell modeling and simulation software environments (e.g. Virtual Cell, E-Cell and CellWare) with their specific ontologies. Virtual Cell ${ }^{30}$ provides a subcellular localization-based visual environment for modeling cellular events. The ontology is mainly based on a single mechanistic physiological model that encodes the general structure and function of a cellular event such as release of calcium and its effects on the cell. In Virtual Cell a cell is considered as distinct geometrical subdomains containing specific cellular components with known concentration. This model allows users to proceed through Virtual Cell simulation tools. Even though Virtual Cell has some applications relevant to GRNs (e.g. Ran-protein dependent transport of proteins between cytosol and nucleus), the platform may have difficulties in modeling events that occur only in one cellular compartment with unknown molecular concentrations.

E-Cell ${ }^{31-32}$ is a generic software platform for visualization, modeling and simulation of whole cell events. E-Cell provides several graphical interfaces for user definable models of certain cellular states. A cell model can be constructed with three classes of objects (entities): substances, genes and reaction rules. The $E$-Cell ontology shares several similarities with the PATIKA ontology which is discussed in the next section.

CellWare $^{33}$ is a multi-algorithmic software platform for modeling and simulation of cellular events. It has several toolboxes including tools for user-dependent model description, definition and construction using a graph editor. A simulation toolbox contains various simulation algorithms and interfaces from which a user can choose. 
Ontology for modeling multi-scale and incomplete networks

The current state of our knowledge on cellular regulatory pathways is still fragmented, incomplete, and uncertain in many respects despite accumulating data. A pathway ontology should be able to represent available information even when it is incomplete, thus allowing incremental construction of pathways. In addition, the ontology must have the flexibility for continuous modification of data without compromising the integrity of the network being built. Therefore the ontology must describe integrity rules of the pathway data, enabling the construction of a robust model of the system. A data integrity rule should state that for every instance of a bioentity (see below), a primary key with an accession number such as (SwissProt ID) must exist and be unique. The seamless integration of various hierarchies of detail or scale is a key problem in modeling and in the representation of complex systems like a cell.

Pathway visualization using diagrams or graphs facilitates the creation of a mathematical model of a GRN. An efficient visualization scheme is generated when an ontology uses intuitive images. The ontology should offer ways to reduce the complexity of the information at some stage of the modeling process.

The discussion in the next sub-section focuses on an ontology that is suitable for modeling incomplete information and abstractions of varying levels of complexity. This ontology has been recently implemented in a pathway database tool named PATIKA (Pathway Analysis Tool for Integration and Knowledge Acquisition). ${ }^{34-35}$ The Pathway Database System (PDS) developed by Krishnamurthy et al. ${ }^{36}$ shares several basic similarities with PATIKA in terms of database organization and visualization. As in PATIKA, PDS provides tools for modeling, storing, analyzing, visualizing and querying 
biological pathways. However, PDS does not define a formal ontology for GRNs but instead follows the rules of $K E G G$ metabolic pathway ontology and uses KEGG data.

An ontology for cellular processes

States and bioentities. Components of a GRN are macromolecules (e.g. DNAs, RNAs or proteins), small molecules (e.g. ions, GTP or ATP), or physical events (e.g. heat, radiation or mechanical stress). Often, these players share a common synthesis pathway and/or are chemically very similar. For example, the p53 protein has many states including its native, phosphorylated, nuclear or MDM2-bound forms. These states are represented as nodes in the network graph, while maintaining their biological or chemical groupings under a common bioentity.

Transitions. A transition represents a cellular event and each is represented as a separate node in the graph (see Fig 4 and Fig 5). A state may go through a certain transition, may be produced by a transition, or may affect a transition as being an activator or inhibitor. When a transition occurs, all of its products are generated. 


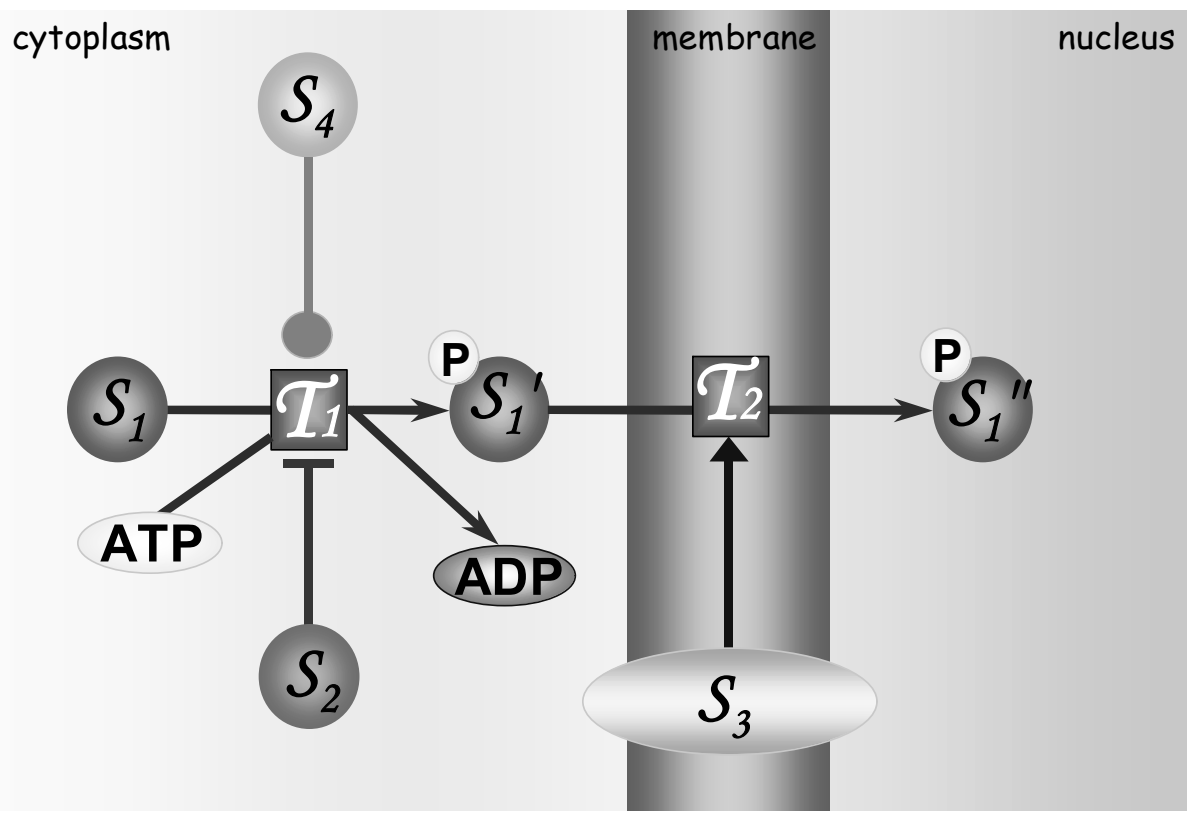

Figure 4. An illustration of the basic features of the PATIKA ontology. States, transitions, and interactions are represented by circles, rectangles and lines, respectively. The bioentity "S," has 3 states (namely, $S_{I}, S_{I}{ }^{\prime}$ and $S_{I}$ ") located in two distinct subcellular compartments (cytoplasm and nucleus) which are separated by a third compartment, the nuclear membrane. $S_{I}$ and $S_{I}{ }^{\prime}$ are both in the cytoplasm. $S_{I}$ is phosphorylated through transition $T_{1}$ giving rise to a new state, the phosphorylated $S_{l}{ }^{\prime}$. $S_{I}$ is translocated to the nucleus through transition $T_{2}$ and becomes $S_{1}{ }^{\prime \prime} . T_{I}$ has two effector states, $S_{2}$ (inhibitor) and $S_{4}$ (unspecified effect). $T_{2}$ has an activator type of effector $\left(S_{3}\right)$ representing, for example, the nuclear pore complex. 


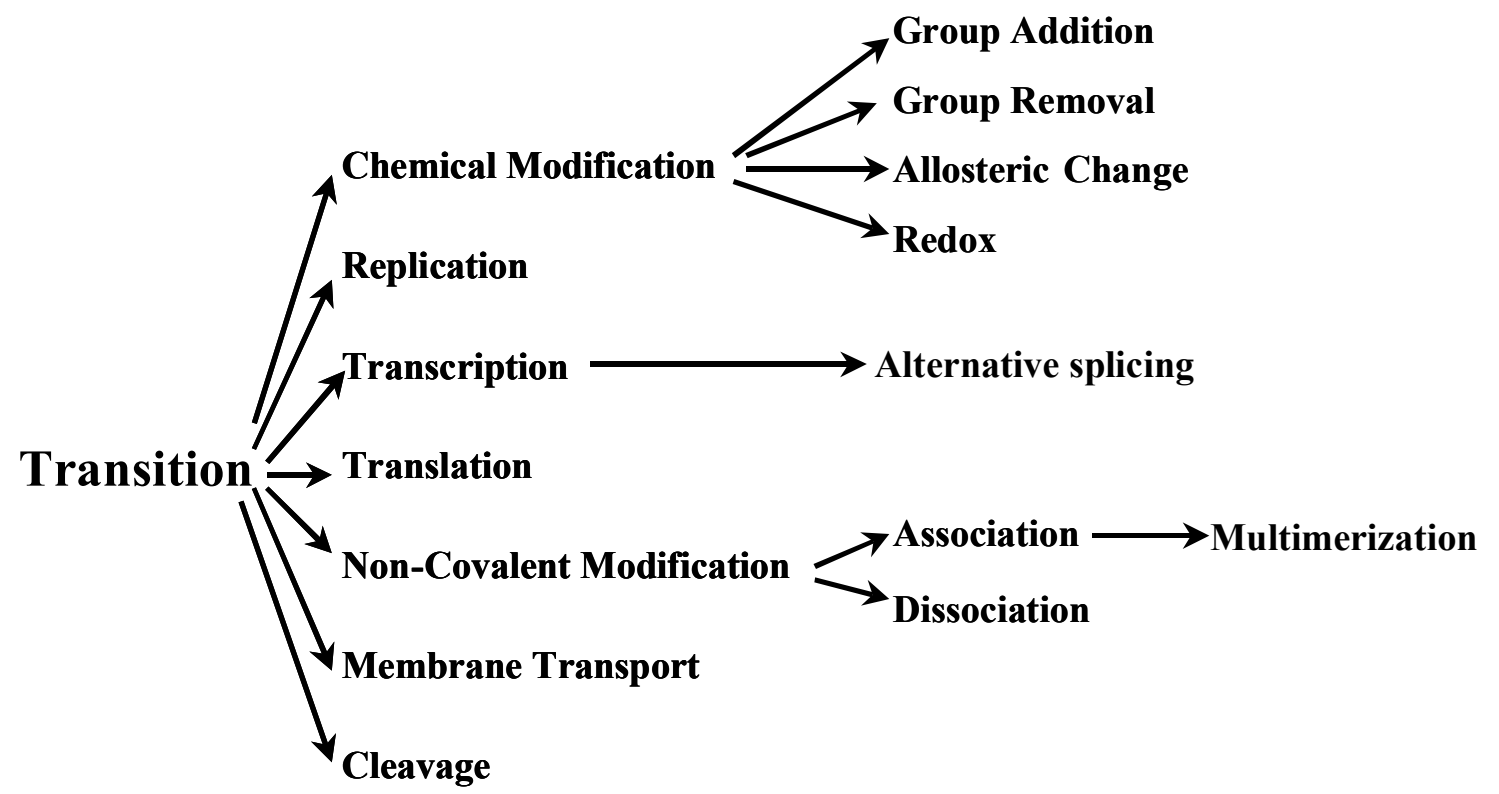

Figure 5. Proposed tree structure used to classify transitions in the PATIKA ontology. If the nature of a transition can not be defined in the existing ontology, it can be considered as generic transition to be defined and added in the ontology. 
Compartments. Transitions also include transport of molecules between cell compartments. The set of transitions that a state can be involved in is strictly related to its compartment; accordingly a change in the compartment means a change in the state's information context. The state's compartment is a part of the ontology. As the compartments and their vicinity are cell-type dependent, compartmental structure can be modeled as part of the ontology. Cell membranes create an additional complexity since not only can a molecule be located completely inside the membrane, it may also communicate with both sides of the membrane as part of the events involved in adjacent compartments. So membranes are considered as separate compartments in the ontology.

Molecular complexes. In biological systems, molecules often form complexes in order to perform certain tasks (Fig 6). Each member of a molecular complex can be considered as a new state of its associated bioentity. The intrinsic specific binding relations affect the function of a molecular complex. Therefore these binding relations must be represented in the model ontology. Moreover, members of a molecular complex may independently participate in different transitions; thus one should be able to address each member individually (Fig 6). In addition, a molecular complex may contain members from neighboring compartments (e.g. receptor-ligand complexes).

Abstractions. Various levels of abstractions are employed in the analysis of complex cellular events. A set of transitions can be described as a single 'process' (e.g. the MAPK pathway), and a set of related processes may be classified under one 'cellular mechanism' (e.g. apoptosis). Some explicit examples of abstractions are shown in Fig 6. In cases where it is not identified which state among a set of states constitutes the 
substrate or effector of a transition, or where target transition of an effector is unclear, we may need to abstract these states (or transitions) as a single state (or transition) to represent the available information despite its incomplete nature.

\section{The PATIKA pathway ontology}

A pathway is an abstraction of a certain biological event and is the primary abstraction in the PATIKA ontology. ${ }^{35}$ The context of this abstraction can change from a single molecule-molecule interaction to a complete network of all the interactions in a cell. In PATIKA, a pathway is represented by a pathway graph, which is a compound graph. ${ }^{37}$ A pathway graph is defined by an interaction graph $G=(V, E)$ along with a number of rules on the topology; $V$ is the union of a finite set of states $V_{\mathrm{s}}$ and a finite set of transitions $V_{\mathrm{t}}$. E is the union of interactions of five sets: substrate edges $E_{\mathrm{s}}$, product edges $E_{\mathrm{p}}$, activator effector edges $E_{\mathrm{a}}$, inhibitor effector edges $E_{\mathrm{i}}$, effector of unknown type edges $E_{\mathrm{u}}$, and each directed edge belonging to either $V_{\mathrm{t}} \times V_{\mathrm{s}}$ (for product edges) or to $V_{\mathrm{s}} \times$ $V_{\mathrm{t}}$ (for remaining interaction edge types). Every state has a defined type: DNA, RNA, protein, small molecule or physical factor. States are also associated with a specific compartment. Identical states in different compartments are considered as separate states. States of the same biological origin and/or similar chemical structure are grouped under a biological entity or simply bioentity that act as state and transition connectivity data holders in PATIKA. 

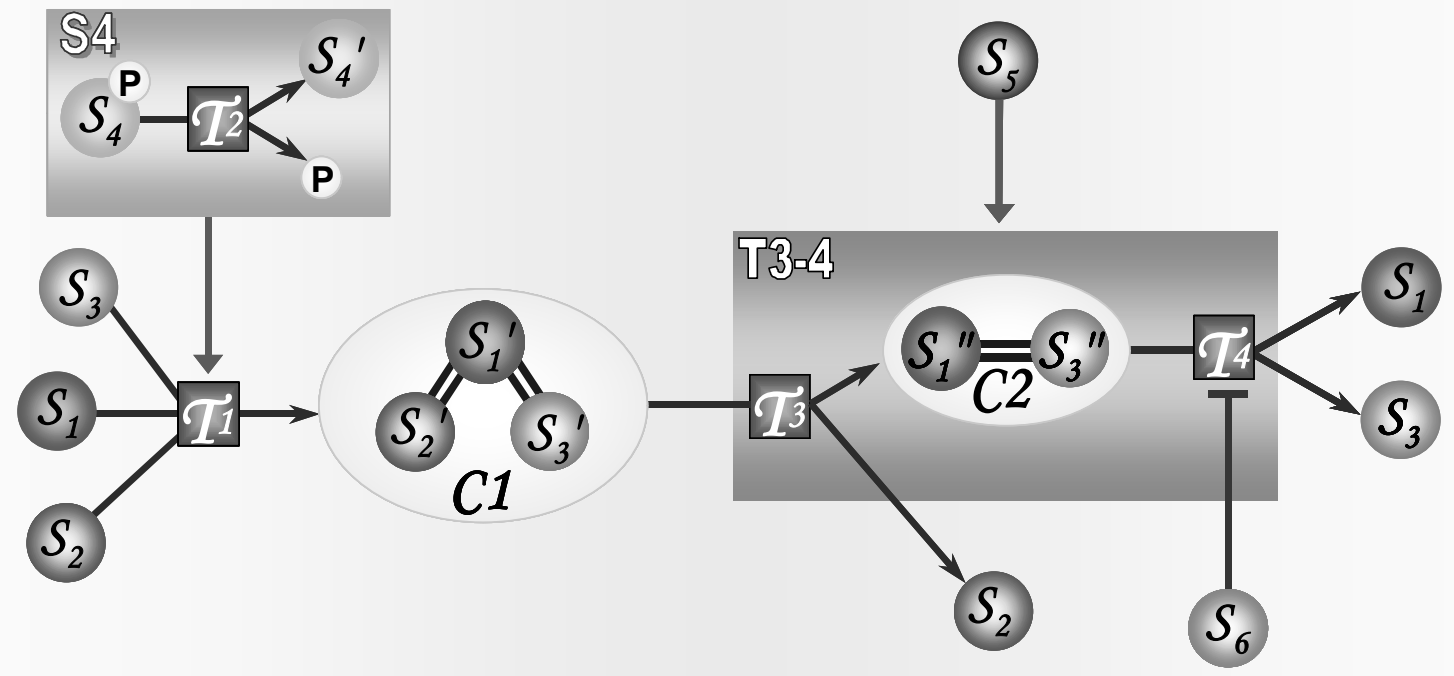

Figure 6. A pathway containing two abstractions and a molecular complex $C 1$ (composed of three states $S_{1}, S_{2}$ and $S_{3}$ ). Super-state $\mathbf{S 4}$ is an example of an abstraction in which the state $S_{4}-\mathrm{P}$ or $\mathrm{S}_{4}$ ' may act as an activator of transition $T_{2} . \quad S_{5}$ leads to the dissociation of complex $C 1$ acting on either before or after the dissociation of $S_{2}$. Therefore $S_{5}$ may be an activator of either $T_{3}$ or $T_{4}$; thus, $S_{5}$ is illustrated as the activator of super-transition T3-4. 
Every transition must be affiliated with at least one substrate and one product edge. It may have an arbitrary number of effectors, a combination of which defines the exact behavior for the transition. Transitions are classified according to the tree shown in Fig 5. A transition is not associated with a specific compartment; instead, its compartment is determined by its interacting states. Different types of molecules (e.g. protein, DNA and RNA) have distinct user interfaces for easier visual discrimination in PATIKA. Compartmental information is also modeled. PATIKA also implements collaborative construction and modification to existing regulatory signaling data on the database. Therefore PATIKA maintains version numbers as part of the ID of each graph object. Thus it is possible that while a user is working on a PATIKA graph locally, others might change the topology and/or properties of states and transitions in the PATIKA database.

\section{EXTRACTING MODELS FROM PATHWAYS DATABASES}

A clear pathway ontology, as discussed in the previous section, will allow systematic methods for extracting GRN models from the interactions stored in a pathways database. The specific model would, of course, depend on the particular biological question being asked. Here, a brief example is given of how a model is extracted from a network of interactions taken from some of the databases listed in Table

5. The work of Aguda and Tang ${ }^{38}$ on the G1 checkpoint of the cell cycle is used as an example. A cell cycle checkpoint is a surveillance mechanism that arrests or slows down cell cycle progression if something goes wrong, e.g. DNA damage. The significance of 
elucidating the control mechanism of the G1 checkpoint lies in the observation that many human cancers are associated with nonfunctional G1 checkpoints.

A qualitative network of the G1-S transition is shown in Fig 7. The network was generated by integrating information from the published literature, including sequence analysis of upstream regulatory regions of genes that are targeted by the E2F transcription factor family. Aguda and Tang ${ }^{38}$ were interested in finding a minimal subnetwork that is sufficient to explain the switching behavior of the G1 checkpoint. The key step towards finding this subnetwork was the hypothesis that there is a core set of interactions with an intrinsic instability that ultimately generates a switching behavior (see refs. 38 and 39 for details; network stability analysis is discussed in the next section). Experimentally, the activity of cyclin E/CDK2 is used as a marker for the entry into the $\mathrm{S}$ phase of the cell cycle. Hence, this minimal set of interactions must include cyclinE/CDK2.

In the network graph shown in Fig 7, the arrows are interpreted as "activation" and the hammerheads as "inhibition". From this qualitative network, a network stability analysis (discussed in the next section) pointed to a core mechanism involving cyclin E/CDK2, Cdc25A, p27Kip1 and their interactions. These interactions involve two coupled positive feedback loops, namely, between the pair (Cdk2/Cyclin E, Cdc25A) and the pair (Cdk2/Cyclin E, p27). This core mechanism was then used as the basis for a more detailed mechanistic model. The dynamics of the model was coded into differential equations and solved in a computer. The computer simulations reproduced the experimentally observed qualitative behavior of the G1 checkpoint. ${ }^{38}$ 
A discussion of the mathematical and computational tools already available for the analysis of GRN models is given in the next section. Most of the models extracted from pathways databases are expected to be qualitative and incomplete in nature; hence the discussion focuses on qualitative network structures and how these structures influence the capacity of the system to exhibit certain dynamical behavior. 


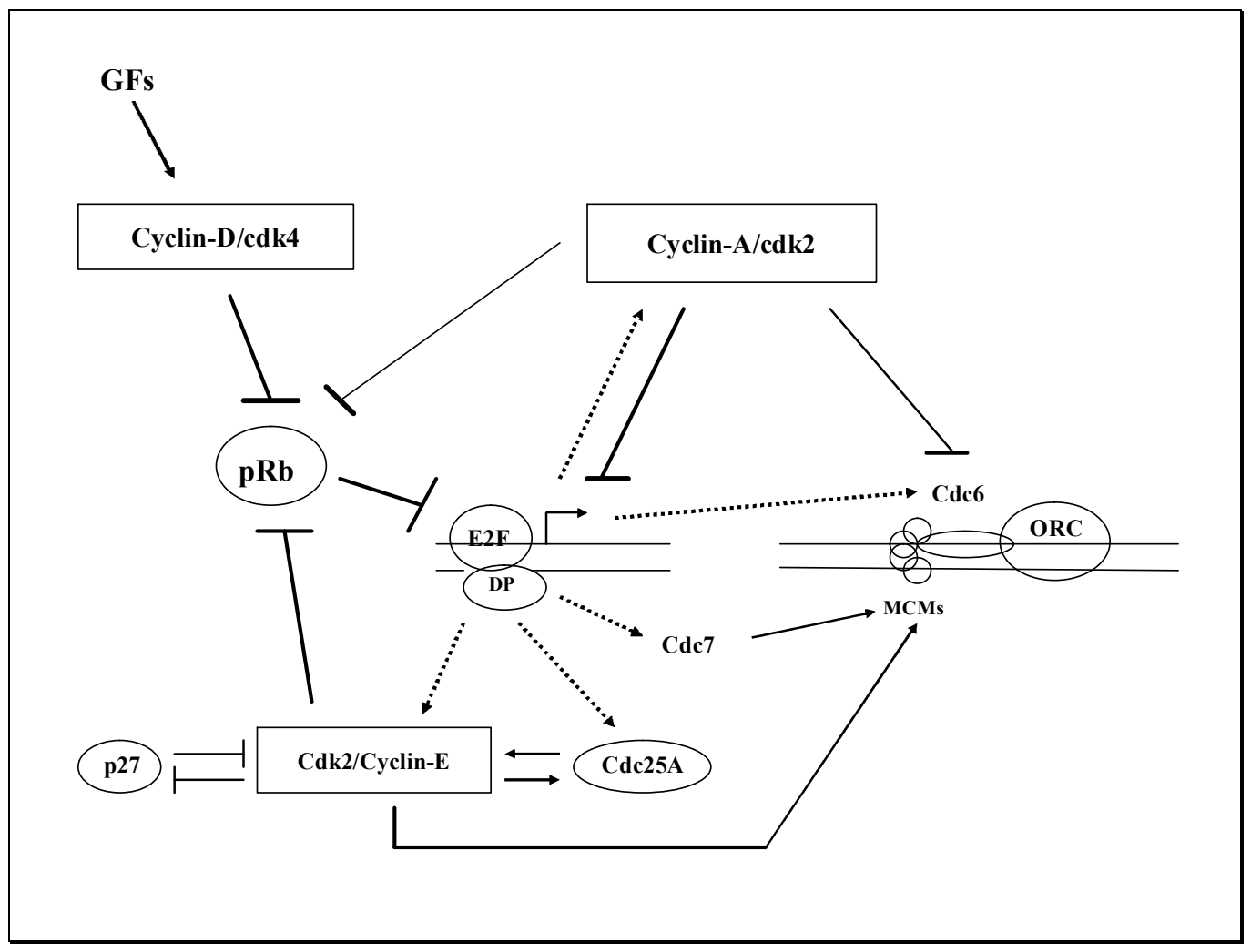

Figure 7. A qualitative network involving key interactions in the G1-S transition of the mammalian cell cycle. Solid lines are post-translational modifications or protein-protein interactions. Dashed arrows are transcriptional steps. Arrows mean "activation", and hammerheads mean "inhibition". GFs = growth factors, $c d k=$ cyclin-dependent kinase, $\mathrm{pRb}=$ retinoblastoma protein, $\mathrm{ORC}=$ origin recognition complex. 


\section{PATHWAY AND DYNAMIC ANALYSIS TOOLS FOR GRNS}

Selection of the appropriate network analysis tool depends on the questions being asked and the scale or size of the network being considered. Questions of robustness of the entire system against perturbations require more consideration of global network properties and less of the attributes of individual processes or reactions. Questions focusing on particular phenomena, such as the switching behavior of a particular set of genes, may require more attention to the local network details involving these genes. How the global and local network properties interplay to produce local or system-level behavior is an important problem that requires multi-scale analysis, both in time and space. In this section, a brief account is given on global network properties, how large networks can be analyzed or reduced by identifying recurring network motifs and extreme pathways, and how topology or network structure alone may already determine a network's stability and its capacity to exhibit certain dynamical behavior. The goal of this section is not to provide a comprehensive review of the aforementioned topics (as they are quite broad and recent reviews will be cited) but, instead, to point out particular directions of analysis of a GRN model once it has been constructed.

\section{Global network properties}

Considering the very large number of interacting genes, proteins and other molecules in a living cell, one would first like to ask questions about global features and properties of the entire network. How connected are the nodes in the network, and what is the mean path length between any two nodes? Are there clusters of interactions so that 
one may subdivide the network into modules? How robust is the system to perturbations - i.e. are there redundant pathways that could take over if a pathway is cut off, so that the system's function is still intact? In general, the aim is to identify global network topological features that affect system function or behavior independent of the details of the individual nodes or interactions. There had been various attempts at searching for quantifiable structural features of metabolic networks, signaling networks, and GRNs (see Ref. 40 for a review). Some basic network descriptors are the degree distribution, the path length distribution, and the clustering coefficient.

The degree distribution $P(k)$ is the probability that a node is linked to $k$ other nodes. The $P(k)$ of random networks exhibits a Poisson distribution whereas that of scale-free networks approximates a power law of the form $P(k) \sim k^{-\gamma}$. An interesting suggestion is that most cellular networks approximate a scale-free topology ${ }^{41-42}$ with an exponent $\gamma$ between 2 and $3 .^{43-44}$ The interpretation of this suggestion is not clear.

The path length distribution of a network tells us how far nodes are from each other. Scale-free networks are 'ultra-small' since they have an average path length of the order $\log (\log N)$, where $N$ is the number of nodes. Random networks are 'small' because their mean path length is of the order $\log N .{ }^{43-44}$

The clustering coefficient of a particular node $A$ of a network is defined by $C(A)$ $=2 n(A) /(k(A)(k(A)-1))$, where $k(A)$ is the number of neighbors of $A$, and $n(A)$ is the number of connections between the neighbors of $A .^{40}$ The average clustering coefficient characterizes the tendency of a network to form node clusters, and is a measure of the network's modularity. The average clustering coefficient of most real networks is larger 
than that of same-size random networks. ${ }^{45}$ Cellular networks have a high average clustering coefficient, which indicates a highly modular structure. ${ }^{46-47}$

\section{Recurring network motifs}

One approach that could simplify the analysis of a large network is to look for recurring motifs which are subgraphs that are over-represented in the network. ${ }^{48-51}$ The motivation is that each motif can be analyzed separately for its intrinsic properties, and the original network may be reduced to a set of motif interactions. Recent analysis ${ }^{48}$ show that three-node feed-forward motifs are abundant in transcriptional regulatory networks and neural networks, while four-node feedback loops are characteristic of electric circuits, but not of biological networks. Remarkable evolutionary conservation of motifs $^{52}$ and convergent evolution toward the same motif types in transcriptional regulatory networks of diverse species ${ }^{53-54}$ show that motifs are indeed significant biologically.

\section{Identifying pathway channels in networks: extreme pathway analysis}

Another way of coping with large networks involves breaking down the network into channels through which distinct processes are carried out. Clarke ${ }^{55}$ developed a formalism called 'Stoichiometric Network Analysis' and was the first to show that all steady-state fluxes are found in a convex set called the 'current cone'; furthermore, he showed that each cone has a certain number of edge vectors that can be uniquely determined from the stoichiometric matrix. Clarke referred to the pathways corresponding to the edge vectors as 'extreme currents'; alternatively, these are called 
extreme pathways in this chapter. Recent algorithms for computing extreme pathways can be found in references 56 and 57. The network shown in Fig 8 serves as an illustration of the basic ideas of extreme pathway analysis. In the network of Fig 8, there are six internal fluxes (labeled $\mathrm{v}_{1}-\mathrm{v}_{6}$ ), and four exchange fluxes (the reversible arrows $b_{1}$ $\mathrm{b}_{4}$ showing exchange across the rectangular boundary). Except for the two cycling pathways corresponding to the two reversible reactions $\left(\mathrm{v}_{2}-\mathrm{v}_{3}\right.$ and $\left.\mathrm{v}_{4}-\mathrm{v}_{5}\right)$, the five extreme pathways are shown in the lower panel of Fig 8. Extreme pathway analysis has been extensively applied to metabolic networks. ${ }^{56-57}$

Network stability analysis

One of the usual purposes of GRN modeling is to determine the origins of switching or threshold behaviors. These behaviors are often associated with the stability properties of the system against perturbations. Would initial perturbations of a species or a reaction in the network die out or would it reverberate throughout the network? It can be shown that, at least near steady states, the stability of the network is influenced by the network structure to a large extent.

More often than not, kinetic or other rate parameters are unknown in GRNs. Only the qualitative interactions between species are usually known, e.g. "X activates $\mathrm{Y}$ " or "V inhibits W". As mentioned earlier, one can interpret the meaning of these qualitative interactions as follows: $\partial(\mathrm{dY} / \mathrm{dt}) / \partial \mathrm{X}>0$, and $\partial(\mathrm{dW} / \mathrm{dt}) / \partial \mathrm{V}<0$, respectively. $\mathrm{A}$ 

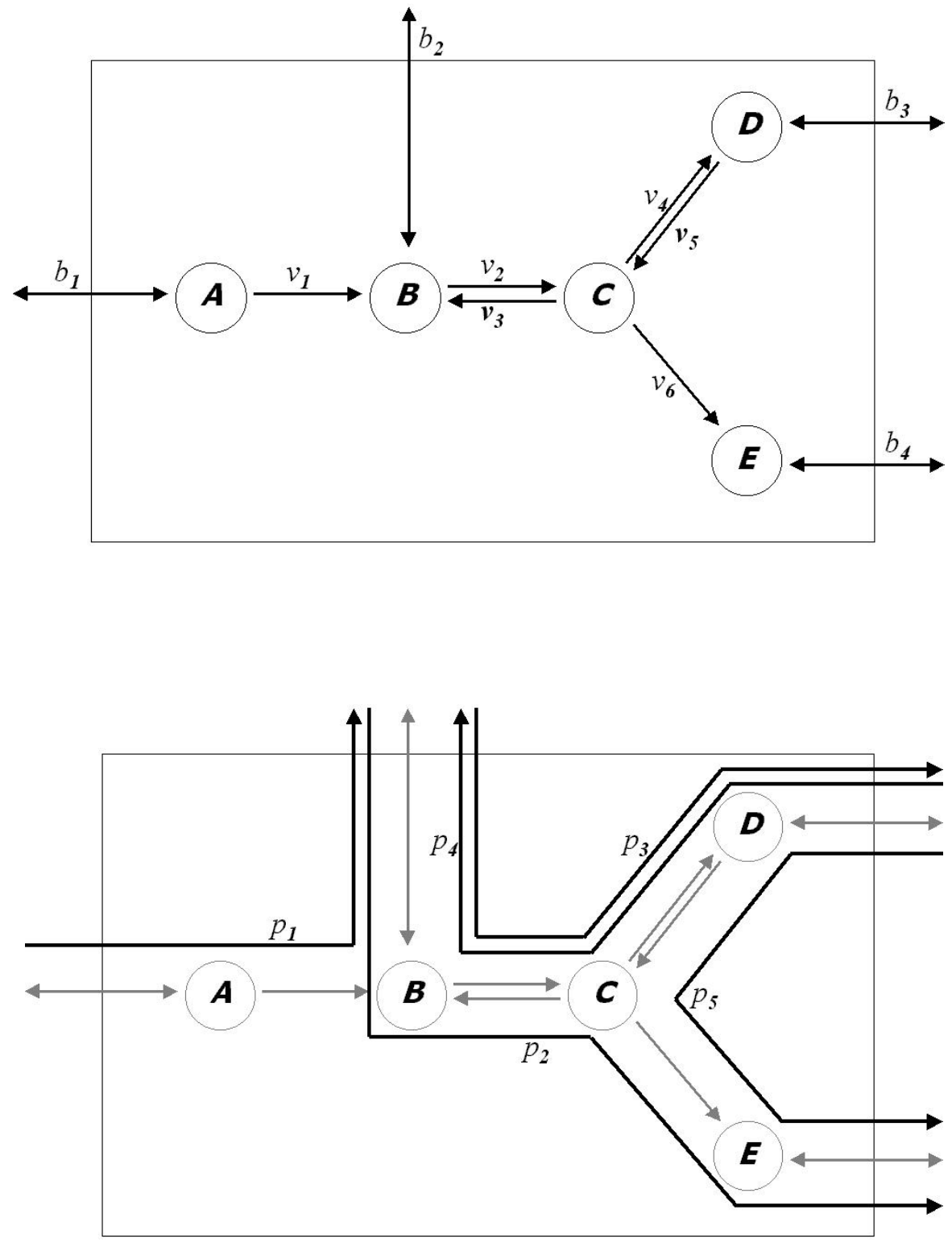

Figure 8. A reaction network and its extreme pathways labeled $p_{1}-p_{5}$ (adapted from Ref. 57) 
'qualitative network' can be defined as a set of nodes (species) and a set of qualitative interactions ('activation' and 'inhibition'). Note that these qualitative interactions are none other than the elements of the Jacobian matrix of a linearized system of differential equations. The state $\boldsymbol{x}$ of a linear dynamical system varies according to the differential equation

$$
d \boldsymbol{x} / d t=\underline{A} \boldsymbol{x}
$$

where $\underline{\boldsymbol{A}}=\left[a_{i j}\right]$ is an $n \times n$ matrix, and $n$ is the number of species. For the case of a biochemical network, $\boldsymbol{x}$ is the vector of perturbations from a steady state. This dynamical system is stable if each solution $\boldsymbol{x}(t)$ approaches zero for $t$ large enough. A weaker condition is that the dynamical system is semistable, which means that, as $t$ becomes larger and larger, the solution $\boldsymbol{x}(t)$ could increase, but not at an exponential rate. It is well known that the dynamical system in equation [4] is stable if and only if all eigenvalues of $\underline{A}$ have negative real parts, and it is semistable if and only if all eigenvalues of $\underline{\boldsymbol{A}}$ have nonpositive real part. The eigenvalues $\lambda$ of the matrix $\underline{\boldsymbol{A}}$ is given by the roots of the characteristic polynomial:

$$
\operatorname{det}(\lambda \underline{\mathbf{I}} \underline{-\mathbf{A}})=\lambda^{\mathrm{n}}+\alpha_{1} \lambda^{\mathrm{n}-1}+\alpha_{2} \lambda^{\mathrm{n}-2}+\ldots+\alpha_{\mathrm{n}-1} \lambda+\alpha_{\mathrm{n}}=0
$$

The coefficients $\alpha_{\mathrm{i}}$ are functions of the elements of $\underline{\boldsymbol{A}}$ and, more importantly, the $\alpha_{\mathrm{i}}$ 's are functions of cycles in the qualitative network graph. ${ }^{58}$ An example of cycles would be the three-cycle $\left(a_{12} a_{23} a_{31}\right)$ and the one-cycle $\left(a_{i i}\right)$. The eigenvalues, and therefore the linear stability of a network, are determined only by cycles in the qualitative network graph.

Suppose that only the sign pattern of the matrix $\underline{\boldsymbol{A}}$ is known, i.e., the magnitudes of $a_{i j}$ matrix entries are unknown but their algebraic signs are known. If all matrices that 
have the same sign pattern as $\underline{\boldsymbol{A}}$ are stable then $\underline{\boldsymbol{A}}$ is referred to as sign-stable. If all matrices that have the same sign pattern as $\underline{\boldsymbol{A}}$ are semistable then $\underline{\boldsymbol{A}}$ is sign-semistable.

The notion of sign-semistability has a simple characterization in terms of signs of the entries of the matrix $\underline{\boldsymbol{A}}$ (the notion of sign-stability can also be characterized in terms of signs of the entries of the matrix $\underline{\boldsymbol{A}}$, but in a more complicated way ${ }^{59}$ ). A useful theorem ${ }^{60}$ states that $\underline{\boldsymbol{A}}$ is sign-semistable if and only if three conditions are met: (i) there is no excitatory one-cycle, (ii) any two-cycle must be negative (i.e. one edge must be inhibitory and the other excitatory), and (iii) there are no cycles of length three. Note that since lack of sign-semistability implies lack of sign-stability, the theorem ${ }^{60}$ on signsemistability also gives a set of necessary conditions for sign-stability.

Predicting dynamics and bistability from network structure alone

It will be useful to identify or classify classes of network structures that, from their structures alone, it is possible to tell whether they have the capacity to exhibit certain behavior. Given a biochemical reaction network, one can ask the following question: are there circumstances under which this network would exhibit phenomena like periodic oscillations and/or bistability? For example, one would want to know the answer to this question when modeling the cell division cycle and circadian rhythm where periodic oscillations are required. For mass-action kinetics models, an extensive theoretical work already exists that answers this type of question for large classes of reaction networks. One such set of results is the deficiency theory. ${ }^{61-64}$ The deficiency $\delta$ of a reaction network is a function of the number of objects and linkages in the network and can be computed easily even if the rate expressions and kinetic parameters are 
unknown. For reaction networks with $\delta=0$ it was shown that they do not have the capacity to exhibit cyclic variation or bistability. ${ }^{63}$ Feinberg also showed that some networks with deficiency $\delta>0$ also do not have the capacity for bistability, if they have some additional properties. ${ }^{64}$ These methods are implemented in the software package called Chemical Reaction Network Toolbox. ${ }^{64-65}$ Recently, other methods of deciding on the capacity for bistability of biochemical reaction networks were developed. ${ }^{66-67}$ The $S R$ graph method of Craciun and Feinberg ${ }^{67}$ allows one to draw conclusions on the capacity of a network to exhibit bistability based on the properties of cycles in the graph.

\section{CONCLUDING REMARKS}

With a well defined pathway ontology, one could envisage a computer program that automates the analysis of complex gene regulatory networks and the extraction or building of GRN models; these models can then be analyzed by computer simulation and other mathematical methods. An investigator would most likely start with a short list of genes or even a short list of specific cellular processes (from which a gene list could be derived using existing gene annotations such as GeneOntology). By scouring databases, the computer program would then try to establish pathways among the initial set of genes; this step will increase the number of genes in the network and also include proteins and other molecules regulating the pathways. At this point, the GRN is a static graph, perhaps a qualitative network containing some information about how the nodes affect each other. The computer program can now use network analysis tools to study the 
topology of the GRN and to identify stabilizing or destabilizing cycles, extreme pathways, or even try to reduce the size of the network without removing the capacity for certain behaviors of interest. Databases (including the published literature) containing experimental information will have to be consulted to validate the significance or strength of contribution of the pathways and cycles present in the reduced model. The rate expressions and associated kinetic parameters of the individual steps in the model are then supplied to a solver of the dynamical equations to simulate the temporal evolution of the model system. Predictions of the model will have to be compared with experimental data, and the process of model refinement and experimental validation could be iterated.

As the work of Ozbudak et al. ${ }^{3}$ and Vilar, Guet and Leibler ${ }^{4}$ on the lac operon demonstrated, abstract kinetic models with a few variables are sometimes sufficient to capture the essential behavior of the system, e.g. the bistable switch in the lac operon. It may seem that there is some arbitrariness in how these simple lac operon models $\mathrm{s}^{3-4}$ were generated, since they seem to look very different and the dynamical variables are not the same. However, both models preserve the common property of having a positive feedback loop. The presence of such a loop has long been known, in dynamical systems theory, to give a system the ability to generate bistability given the right parameters. As the work of Ozbudak et al. ${ }^{3}$ showed, a low-dimensional abstract model can indeed be predictive. In the future, the process of extracting an abstract model from a complex GRN may well be carried out systematically. The key will be the application of the mathematical fields of nonlinear dynamics and reaction network analysis. As mentioned in this chapter, possible behavior of networks may already be predicted from their qualitative network structures regardless of the values of rate parameters. The 
development of a pathway ontology that can interface with network structure analysis tools will be crucial for the integration and use of the huge amounts of data stored in databases.

Significant advances towards understanding gene networks are coming from recent work on synthetic gene networks (see ref. 68 for a recent review); the goal here is the construction and engineering control of genetic circuits built from well understood building blocks of small gene modules. What is being learned from these man-made gene networks will be very useful in future analysis of the very complex GRN repertoire of a living cell. 


\section{REFERENCES}

1. P. Wong, S. Gladney, and J. D. Keasling, Biotechnol. Prog., 13, 132 (1997).

Mathematical Model of the Lac Operon: Inducer Exclusion, Catabolite Repression, and Diauxic Growth on Glucose and Lactose.

2. N. Yildirim and M. C. Mackey, Biophysical Journal, 84, 2841 (2003). Feedback Regulation in the Lactose Operon: A Mathematical Modeling Study and Comparison with Experimental Data.

3. E. M. Ozbudak, M. Thattai, H. N. Lim, B. I. Shraiman, and A. van Oudenaarden, Nature, 427, 737 (2004). Multistability in the Lactose Utilization Network of Escherichia coli.

4. J. M. G. Vilar, C C. Guet, and S. Leibler, J. Cell Biology, 161, 471 (2003). Modeling Network Dynamics: The Lac Operon, A Case Study.

5. F. Jacob, D. Perrin, C. Sanchez, and J. Monod, Compt. Rendu. Acad. Sci., 245, 1727 (1960). L'operon: Groupe de Genes a l'Expression Coordonne par un Operateur.

6. J. J. Tyson and H. G. Othmer, in Progress in Biophysics, R. Rosen, Ed., Academic Press, New York, 5, 1 (1978). The Dynamics of Feedback Control Circuits in Biochemical Pathways. 
7. D. M. Wolf and F. H. Eeckman, J. Theor. Biol., 195, 167 (1998). On the Relationship between Genomic Regulatory Element Organization and Gene Regulatory Dynamics.

8. J. J. Tyson, K. C. Chen, and B. Novak, Curr. Opin. Cell Biol., 15, 221 (2003).

Sniffers, Buzzers, Toggles and Blinkers: Dynamics of Regulatory and Signaling Pathways in the Cell.

9. H. de Jong, J. Comput. Biol., 9, 67 (2002). Modeling and Simulation of Genetic Regulatory Systems: A Literature Review.

10. N. Friedman, Science, 303, 799 (2004). Inferring Cellular Networks Using Probabilistic Graphical Models.

11. E. P. van Someren, L. F. A. Wessels, E. Backer, and M. J. T. Reinders, Pharmacogenomics, 3, 507 (2002). Genetic Network Modeling.

12. J. Stark, D. Brewer, M. Barenco, D. Tomescu, R. Callard, and M. Hubank, Biochem. Soc. Trans., 31, 1519 (2003). Reconstructing Gene Networks: What are the Limits?

13. J. Stark, R. Callard, and M. Hubank, Trends Biotech., 21, 290 (2003). From the Top Down: Towards a Predictive Biology of Signaling Networks. 
14. P. D’haeseleer, S. Liang, and R. Somogyi, Bioinformatics, 16, 707 (2000). Genetic Network Inference: From Co-expression Clustering to Reverse Engineering.

15. M. B. Eisen, P. T. Spellman, P. O. Brown, and D. Botstein, Proc. Natl. Acad. Sci. USA, 95, 14863 (1998). Cluster Analysis and Display of Genome-Wide Expression Patterns.

16. J. Wang, O. Myklebost, and E. Hovig, Bioinformatics, 19, 2210 (2003). MGraph: Graphical Models for Microarray Data Analysis.

17. D. Husmeier, Biochem. Soc. Trans., 31, 1516 (2003). Reverse Engineering of Genetic Networks with Bayesian Networks.

18. D. Husmeier, Bioinformatics, 19, 2271 (2003b). Sensitivity and Specificity of Inferring Genetic Regulatory Interactions from Microarray Experiments with Dynamic Bayesian Networks.

19. N. Friedman, M. Linial, I. Nachman, and D. Pe'er, J. Comput. Biol., 7, 601 (2000). Using Bayesian networks to Analyze Expression Data.

20. D. Pe'er, A. Regev, G. Elidan, and N. Friedman, Bioinformatics, 17, S215 (2001). Inferring Subnetworks from Perturbed Expression Profiles. 
21. A. J. Hartemink, D. K. Gifford, T. S. Jaakkola, and R. A. Young, Pac. Symp. Biocomput., 6, 422 (2001). Using Graphical Models and Genomic Expression Data to Statistically Validate Models of Genetic Regulatory Networks.

22. D. E. Zak, F. J. Doyle, and J. S. Schwaber, Proceedings of the Third International Conference on Systems Biology, Karolinska Institute, Sweden, pp. 236-237 (2002). Local Identifiability: When can Genetic Networks be Identified from Microarray Data?

23. A. de la Fuente, P. Brazhnik, and P. Mendes, Trends Genet., 18, 395 (2002). Linking the Genes: Inferring Quantitative Gene Networks from Microarray Data.

24. B. N. Kholodenko, A. Kiyatkin, F. J. Bruggeman, E. Sontag, H. V. Westerhoff, and J. B. Hoek, Proc. Natl. Acad. Sci. USA, 99, 12841 (2002). Untangling Wires: A Strategy to Trace Functional Interactions in Signaling and Gene Networks.

25. T. S. Gardner, D. di Bernardo, D. Lorenz, and J. J. Collins, Science, 301, 102 (2003). Inferring Genetic Networks and Identifying Compound Mode of Action via Expression Profiling.

26. M. Y. Galperin, Nucleic Acids Res., 32, D3 (2004). The Molecular Biology Database Collection: 2004 update. 
27. C. Lemer, E. Antezana, F. Couche, F. Fays, X. Santolaria, R. Janky, Y. Deville, J Richelle, and S.J. Wodak, Nucleic Acids Res., 32, D443 (2004). The aMAZE LightBench: A Web Interface to a Relational Database of Cellular Processes.

28. T. R. Gruber. Knowledge Acquisition, 5, 199 (1993). A Translation Approach to Portable Ontologies.

29. P. Mendes, Trends Biochem. Sci., 22, 361 (1997). Biochemistry by Numbers: Simulation of Biochemical Pathways with Gepasi 3.

30. B. M. Slepchenko, J. C. Schaff, I. Macara, and L. M. Loew, Trends Cell Biol., 13, 570 (2003). Quantitative Cell Biology with the Virtual Cell.

31. M. Tomita, K. Hashimoto, K. Takahashi, T. Shimizu, Y. Matsuzaki, F. Miyoshi, K. Saito, S. Tanida, K. Yugi, J. C. Venter, and C. A. Hutchison, Bioinformatics., 15, 72 (1999). E-CELL: Software Environment for Whole-Cell Simulation.

32. K. Takahashi, N. Ishikawa, Y. Sadamoto, H. Sasamoto, S. Ohta, A. Shiozawa, F. Miyoshi, Y. Naito, Y. Nakayama, and M. Tomita, Bioinformatics., 19, 1727 (2003). ECell 2: Multi-Platform E-Cell Simulation System.

33. P. Dhar, T.C. Meng, S. Somani, L. Ye, A. Sairam, M. Chitre, Z. Hao, and K. Sakharkar, Bioinformatics., 20, 1319 (2004). Cellware: A Multi-Algorithmic Software for Computational Systems Biology. 
34. E. Demir, O. Babur, U. Dogrusoz, A. Gursoy, G. Nisanci, R. Cetin-Atalay, and M. Ozturk, Bioinformatics., 18, 996 (2002). PATIKA: An Integrated Visual Environment for Collaborative Construction and Analysis of Cellular Pathways.

35. E. Demir, O. Babur, U. Dogrusoz, A. Gursoy, A. Ayaz, G. Gulesir, G. Nisanci, and R. Cetin-Atalay, Bioinformatics., 20, 349 (2004). An Ontology for Collaborative Construction and Analysis of Cellular Pathways.

36. L. Krishnamurthy, J. Nadeau, G. Ozsoyoglu, M. Ozsoyoglu, G. Schaeffer, M. Tasan, and W. Xu, Bioinformatics., 22, 930, (2003). Pathways Database System: An Integrated System for Biological Pathways.

37. K. Fukuda and T. Takagi, Bioinformatics, 17, 829 (2001). Knowledge Representation of Signal Transduction Pathways.

38. B. D. Aguda and Y. Tang, Cell Proliferation, 32, 321 (1999). The Kinetic Origins of the Restriction Point in the Mammalian Cell Cycle.

39. B. D. Aguda, Oncogene 18, 2846 (1999). Instabilities in Phosphorylation Dephosphorylation Cascades and Cell Cycle Checkpoints.

40. A.-L. Barabasi and Z. N. Oltvai, Nat. Rev. Genet., 5, 101 (2004). Network Biology: Understanding the Cell's Functional Organization. 
41. H. Jeong, B. Tombor, R. Albert, Z. N. Oltvai, and A.-L. Barabasi, Nature, 407, 651 (2000). The Large-Scale Organization of Metabolic Networks.

42. A. Wagner and D. A. Fell, Proc. R. Soc. Lond. B, 268, 1803 (2001). The Small World Inside Metabolic Networks.

43. F. Chung and L. Lu, Proc. Natl. Acad. Sci. USA, 99, 15879 (2002). The Average Distances in Random Graphs with Given Expected Degrees.

44. R. Cohen and S. Havlin, Phys. Rev. Lett., 90, 058701 (2003). Scale-Free Networks are Ultra Small.

45. D. J. Watts and S. H. Strogatz, Nature, 393, 440 (1998). Collective Dynamics of 'Small-World' Networks.

46. A. Wagner, Mol. Biol. Evol., 18, 1283 (2001). The Yeast Protein Interaction Network Evolves Rapidly and Contains Few Redundant Duplicate Genes.

47. S. Wuchty, Mol. Biol. Evol., 18, 1694 (2001). Scale-Free Behaviour in Protein Domain Networks.

48. R. Milo, S. S. Shen-Orr, S. Itzkovitz, N. Kashtan, D. Chklovskii, and U. Alon, Science, 298, 824 (2002). Network Motifs: Simple Building Blocks of Complex Networks. 
49. S. Itzkovitz, R. Milo, N. Kashtan, G. Ziv, and U. Alon, Phys. Rev. E Stat. Nonlin. Soft Matter Phys., 68, 026127 (2003). Subgraphs in Random Networks.

50. E. Yeger-Lotem, S. Sattath, N. Kashtan, S. Itzkovitz, R. Milo, R. Y. Pinter, and U. Alon, Proc. Natl. Acad. Sci. USA, 101, 5934 (2004). Network Motifs in Integrated Cellular Networks of Transcription-Regulation and Protein-Protein Interaction.

51. J. Ihmels, G. Friedlander, S. Bergmann, O. Sarig, Y. Ziv, and N. Barkai, Nature Genet., 31, 370 (2002). Revealing Modular Organization in the Yeast Transcriptional Network.

52. S. Wuchty, Z. N. Oltvai, and A.-L. Barabasi, Nature Genet., 35,176 (2003). Evolutionary Conservation of Motif Constituents within the Yeast Protein Interaction Network.

53. G. C. Conant and A. Wagner, Nature Genet., 34, 264 (2003). A Convergent Evolution of Gene Circuits.

54. V. F. Hinman, A. T. Nguyen, R. A. Cameron, E. H. Davidson, Proc. Natl. Acad. Sci. USA, 100(23), 13356 (2003). Developmental Gene Regulatory Network Architecture Across 500 Million Years of Echinoderm Evolution. 
55. B. L. Clarke, $A d v$. Chem. Phys., 43, 1 (1980). Stability of Complex Reaction Networks.

56. J. A. Papin, N. D. Price, and B. Ø. Palsson, Genome Res., 12, 1889 (2000). Extreme Pathway Lengths and Reaction Participation in Genome-Scale Metabolic Networks.

57. C. Schilling, D. Letscher, and B. Ø. Palsson, J. Theor. Biol., 203, 229 (2000). Theory for the Systemic Definition of Metabolic Pathways and their use in Interpreting Metabolic Function from a Pathway-Oriented Perspective.

58. C. J. Puccia and R. Levins, Qualitative Modeling of Complex Systems: An Introduction to Loop Analysis and Time Averaging. Harvard University Press, Cambridge, Mass., 1985.

59. V. Klee, in Applications of Combinatorics and Graph Theory to the Biological and Social Sciences, F. Roberts, Ed., IMA Volumes in Mathematics and Its Applications, Springer, New York, 17, 1989, pp. 203-219. Sign-Patterns and Stability.

60. J. Quirk and R. Ruppert, Rev. Economic Studies, 32, 311 (1965). Qualitative Economics and the Stability of Equilibrium. 
61. M. Feinberg, Arch. Rational Mech. Anal., 49, 187 (1972). Complex Balancing in General Kinetic Systems.

62. F. Horn, R. Jackson, Arch. Rational Mech. Anal., 47, 81 (1972). General Mass Action Kinetics.

63. M. Feinberg, Lectures on Chemical Reaction Networks, written version of lectures given at the Mathematical Research Center, University of Wisconsin, Madison. Mathematical Research Center, University of Wisconsin, 1979.

64. M. Feinberg, Arch. Rational Mech. Anal., 132, 311, (1995). The Existence and Uniqueness of Steady States for a Class of Chemical Reaction Networks.

65. M. Feinberg, Chemical Reaction Network Toolbox, Version 1.02 (1995), and Version 1.1 (1999, with P. Ellison) available for download at http:// www.che.eng.ohiostate.edu/ feinberg/crnt

66. G. Craciun and M. Feinberg, SIAM J. Appl. Math., in press (2004). Multiple Equilibria in Complex Chemical Reaction Networks: The Injectivity Property.

67. G. Craciun and M. Feinberg, Mathematical Biosciences Institute Technical Report, 22 (2004). Multiple Equilibria in Complex Chemical Reaction Networks: The SR Graph. 
68. R. Weiss, S. Basu, S. Hooshangi, A. Kalmbach, D. Karig, R. Mehreja, and I. Netravali, Natural Computing, 2, 47 (2003). Genetic Circuit Building Blocks for Cellular Computation, Communications, and Signal Processing. 